Caracterización del estado fundamental del Hamiltoniano asociado al modelo del parámetro de impacto.

Héctor Merino Cruz

Director de tesis:

Dr. Juan Héctor Arredondo Ruiz

México, D. F., Febrero de 2009. 


\title{
Caracterización del estado fundamental del Hamiltoniano asociado al modelo del parámetro de impacto.
}

\author{
Héctor Merino Cruz
}

México, D. F., Febrero de 2009 


\section{Agradecimientos}

Agradezco a muchas personas que estuvieron conmigo durante esta etapa de mi vida académica.

Entre ellos los revisores, los doctores Antoni Wawrzynczyk (UAM-I), Fco. Javier Mendoza (BUAP), Rafael del Río (IIMAS-UNAM) y, mi director de tesis, Juan Héctor Arredondo (UAM-I). Sus observaciones permitieron mejorar varios aspectos de este trabajo. De manera especial agradezco a Juan Héctor Arredondo por la comprensión, ayuda y paciencia que me mostró en todo este tiempo que estuvo en la dirección de este trabajo.

Agradezco también a los profesores Shirley Bromberg, Luis Aguirre, Peter Seibert y Roberto Quezada, por todo el apoyo que me brindaron durante mi estancia en la UAM.

Una de las personas que siempre estuvo a mi lado dándome aliento a no desistir es mi esposa. Muchas gracias Silvia.

Finalmente, agradezco por el financiamiento que se me otorgó a través del Conacyt en el programa de becas para estudio de posgrado. 


\section{Índice general}

Agradecimientos I

$\begin{array}{ll}\text { Introducción } & 1\end{array}$

1. Teoría básica para el análisis de operadores 5

1.1. La transformada de Fourier . . . . . . . . . . . 5

1.2. Teorema Espectral y Teorema de Stone . . . . . . . . 9

1.3. Espectro esencial y teorema de Weyl . . . . . . . . 15

1.4. Convergencia de operadores no acotados . . . . . . 16

2. Estudio del espectro discreto de un hamiltoniano dependiente del tiempo asociado al modelo del parámetro de impacto

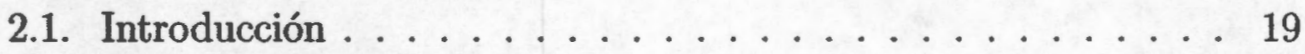

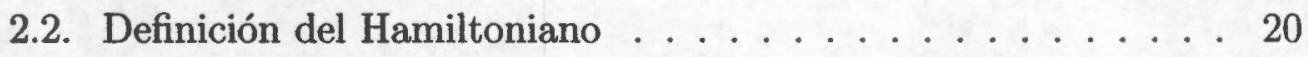

2.3. Interpretación física . . . . . . . . . . . . 21

2.4. Espectros de operadores asociados . . . . . . . . . . 21

2.5. Caracterización del espectro discreto del hamiltoniano . . . . 24

2.6. Caracterización del estado fundamental . . . . . . . . 30

3. Sobre los valores propios de un operador dependiente del tiempo

3.1. La definición del operador . . . . . . . . . . 34

3.2. El teorema . . . . . . . . . . . . . . 35

3.3. La demostración . . . . . . . . . . . . . . 36

3.4. Resultado principal. La dimensión de la proyección espectral sobre $\left[-E_{0},-E_{\infty}\right]$ y el vector propio correspondiente $\ldots \ldots 46$ 
Conclusiones

51

Bibliografía

52 


\section{Introducción}

La Mecánica Cuántica estudia el comportamiento de la materia a escala muy pequeña, concretamente, a escalas en las que no es posible determinar con exactitud, de manera simultanea, la posición y el momento de una partícula (contrario al campo de estudio de la Mecánica Clásica o Newtoniana, en dónde ésto si es posible). En Mecánica Cuántica, el conjunto de los estados posibles en un sistema se representa por un espacio de Hilbert complejo $\mathscr{H}$, donde cualquier estado se describe por un vector unitario en este espacio. Una observable (propiedad del estado de un sistema que puede ser determinada por alguna secuencia de operaciones físicas, por ejemplo, energías, posición, momento) se representa por un operador autoadjunto $A$ definido sobre un subespacio denso de $\mathscr{H}$. El valor esperado de una observable $A$ en un estado $\varphi$ es el número real $(\varphi, A \varphi)$. En la representación de Schrödinger la evolución de un sistema cuántico se especifica indicando cómo cambian los estados en el transcurso del tiempo. Dicho movimiento se realiza mediante una familia de operadores unitarios $U(t): \mathscr{H} \rightarrow \mathscr{H}$ generada por un operador autoadjunto $H$ (según el Teorema de Stone) que se denomina Hamiltoniano del sistema.

Un problema clásico en la teoría espectral de operadores lineales es la caracterización de los vectores propios y/o los valores propios asociados a un operador. Este problema ha probado ser bastante difícil en general. De hecho, no existe método alguno para su solución y unicamente los métodos perturbativos ofrecen una respuesta parcial a la caracterización de los eigenvalores. En el caso en que se tiene una familia de operadores dependiente de un parámetro, el problema se complica aún más. A falta de una teoría general es particularmente interesante considerar ejemplos que puedan resolverse exactamente para poder inducir propiedades generales. Éste es uno de los objetivos de este trabajo. Consideramos un Hamiltoniano dependiente del tiempo para el cual se caracteriza el valor propio asociado al estado 
fundamental, siguiendo técnicas empleadas en otros trabajos, principalmente [Arr1] y [AS].

En el artículo de Arredondo[Arr1], se estudia el modelo del parámetro de impacto para la dispersión de una particula ligera y dos partículas pesadas. En particular, se analiza el comportamiento asintótico de la probabilidad de transición cuando la velocidad relativa de las partículas pesadas tienden a cero. En la primera parte de este trabajo, estudiamos el hamiltoniano dependiente del tiempo asociado a este problema. En el modelo que mencionamos, se asume que cada partícula pesada tiene masa muy grande y que sus movimientos a lo largo de una trayectoria clásica, no se afectan por la partícula ligera. En tal aproximación, la influencia de cada partícula pesada sobre la ligera se representa por potenciales dependientes del tiempo. En este trabajo se desprecian los efectos magnéticos y se toman los potenciales de interacción entre la partícula ligera y las pesadas, como separables.

Sobre el espacio de las funciones complejas de cuadrado integrables (respecto a la medida de Lebesgue $d x$ ) sobre $\mathbb{R}^{n}$, denotado por $L^{2}\left(\mathbb{R}^{n}\right)$, se define para cada $t \in \mathbb{R}$, el operador $H_{v}(t)$ con dominio $H^{2}\left(\mathbb{R}^{n}\right)$ (el espacio de Sobolev de orden 2), con

$$
H_{v}(t)=-\frac{1}{2} \Delta-\lambda_{1} V-\lambda_{2} V_{\rho}
$$

donde $\Delta$ es el operador de Laplace, $\Delta:=\frac{\partial^{2}}{\partial x_{1}^{2}}+\cdots+\frac{\partial^{2}}{\partial x_{n}^{2}}$. Las constantes, $\lambda_{1}, \lambda_{2} \in \mathbb{R}^{+}$y $\lambda_{1}>\lambda_{2}$. El operador $V$ esta definido por

$$
V \varphi=(g, \varphi) g, \quad \forall \varphi \in L^{2}\left(\mathbb{R}^{n}\right),
$$

donde $g$ es una función no nula en $L^{2}\left(\mathbb{R}^{n}\right),(f, g)=\int_{\mathbb{R}^{n}} \overline{f(x)} g(x) d x$ denota el producto escalar en $L^{2}\left(\mathbb{R}^{n}\right)$, y el operador $V_{\rho}$ se define como

$$
V_{\rho} \varphi=\left(g_{\rho}, \varphi\right) g_{\rho}, \quad \forall \varphi \in L^{2}\left(\mathbb{R}^{n}\right)
$$

donde

$$
g_{\rho}(\vec{x})=g(\vec{x}-\rho(t))
$$

La función $\rho(t)$ se considera de $\mathbb{R}$ a $\mathbb{R}^{n}$, dos veces continuamente diferenciable y tal que, para algún $t_{ \pm}>0$,

$$
\begin{gathered}
\left|\rho(t)-\overrightarrow{c_{ \pm}}-t \overrightarrow{v_{ \pm}}\right| \leq \frac{C_{0}}{|t|^{\alpha}}, \quad \forall t, \text { con }|t|>t_{ \pm}, \\
\left|\frac{d^{k}}{d t^{k}}\left(\rho(t)-\overrightarrow{c_{ \pm}}-t \overrightarrow{v_{ \pm}}\right)\right| \leq C_{k} v^{k}, \quad \forall t, \text { con }|t|>t_{ \pm}, \text {y para } k=1,2 .
\end{gathered}
$$


En las condiciones anteriores, $\alpha>0, \overrightarrow{c_{ \pm}}, \overrightarrow{v_{ \pm}} \in \mathbb{R}^{n}$ con $\overrightarrow{v_{ \pm}} \neq \overrightarrow{0}$. Además, $v:=\left|\overrightarrow{v_{ \pm}}\right| \leq 1 \mathrm{y}$

$$
\left|\frac{d^{k}}{d t^{k}} \rho(t)\right| \leq v^{k}, \quad \forall t, \text { con }|t|>t_{ \pm}, \text {y para } k=1,2 .
$$

Se asume que $t_{ \pm},\left|\overrightarrow{c_{ \pm}}\right|$y $C_{k}$ están acotadas por un número positivo $M$.

El vector de estado de la partícula ligera satisface la ecuación de Schrödinger

$$
i \frac{\partial}{\partial t} \Psi(t)=H_{v}(t) \Psi(t)
$$

La solución de la ecuación anterior esta dada por una familia de operadores unitarios con dos parámetros

$$
U_{v}(t, s), \quad t, s \in \mathbb{R} .
$$

Sea $H_{1}$ el operador autoadjunto sobre $H^{2}\left(\mathbb{R}^{n}\right)$ definido por

$$
H_{1}=-\frac{1}{2} \Delta-\lambda_{1} V
$$

El operador $-\frac{1}{2} \Delta$ tiene como espectro esencial el conjunto $[0,+\infty)$. También, $H_{1}$ es positivo ó tiene un único valor propio negativo $-Q_{1}$.

Sea $P_{1}$ el proyector ortogonal sobre el espacio fundamental no degenerado de $H_{1}$. La existencia de operadores de onda (o de Möller)

$$
\Omega_{ \pm}(v)=\mathrm{s}-\lim _{t \rightarrow \pm \infty} U_{v}(0, t) e^{-i t H_{1}} P_{1}
$$

se prueba en [Arr1], donde s - lim indica convergencia fuerte. Sea $\phi$ un estado fundamental normalizado de $H_{1}$. Asumiendo que inicialmente la partícula ligera se encuentra en el estado dado por $\phi$, se tiene que la probabilidad que después de la interacción la partícula ligera permanezca en el estado $\phi$ está dado por

$$
P(v)=\left|\left(\phi, \Omega_{+}(v)^{*} \Omega_{-}(v) \phi\right)\right|^{2}=\left|\left(\Omega_{+}(v) \phi, \Omega_{-}(v) \phi\right)\right|^{2} .
$$

En [Arr1] se prueba que para velocidades $v(\approx O)$ pequeñas, la posibilidad de una transición de estado es improbable:

$$
\lim _{v \rightarrow 0} P(v)=1
$$


Este resultado es una justificación teórica rigurosa del criterio de Massey en física experimental.

Éste trabajo está dividido en tres capítulos. En el primero se recogen los conceptos y resultados básicos que usaremos. La mayor parte de los resultados no son demostrados porque los consideramos clásicos y pueden leerse en los libros usuales de Análisis Funcional.

En el capítulo 2 justificamos partes importantes de lo que hemos mencionado al inicio de la introducción, estudiando el hamiltoniano definido en (1), asociado a este problema. Caracterizamos principalmente el estado fundamental y los eigenvalores asociados.

En el capítulo 3 se estudia un hamiltoniano más complicado que el estudiado en el capítulo 2. El hamiltoniano que se estudia en el capítulo 3 está definido de la siguiente manera: Para $t \in \mathbb{R}$, sea

$$
H(t)=-\frac{1}{2} \Delta-\lambda_{1} V_{1}-\lambda_{2} V_{1, \rho}-\mu_{1} V_{2}-\mu_{2} V_{2, \rho},
$$

donde $\Delta$ es el operador de Laplace en $\mathbb{R}^{n}$ y, $\lambda_{i}$ y $\mu_{i}$, con $i=1$, , son constantes positivas. El operador $H(t)$ esta definido sobre $L^{2}\left(\mathbb{R}^{n}, d x\right)$ con dominio $H^{2}\left(\mathbb{R}^{n}\right)$ también. Los operadores $V_{1}$ y $V_{2}$, definidos por

$$
V_{i} \varphi=\left(g_{i}, \varphi\right) g_{i}, \quad \forall \varphi \in L^{2}\left(\mathbb{R}^{n}, d x\right), \quad i=1,2,
$$

y las funciones $g_{i},(i=1,2)$, son funciones no nulas de $L^{2}\left(\mathbb{R}^{n}, d x\right)$.

Además,

$$
V_{i, \rho} \varphi=\left(g_{i, \rho}, \varphi\right) g_{i, \rho}, \quad \text { con } \quad g_{i, \rho}(x)=g_{i}(x-\rho(t)) .
$$

Donde $\rho(t)$ es una función continua de $\mathbb{R}$ a $\mathbb{R}^{n}$, que para simplificar la notación, suponemos que $\rho(0)=\overrightarrow{0} \in \mathbb{R}^{n}$ y

$$
\lim _{|t| \rightarrow \infty}|\rho(t)|=\infty
$$

Como se observa, los hamiltonianos son similares por lo que se espera que mediante técnicas semejantes se puedan obtener y demostrar propiedades que ellos tienen. Así es como caracterizamos también los eigenvalores asociados al estado fundamental del hamitoniano (2) y obtenemos un un resultado original. Mostramos que el estado fundamental de $H(t)$ es no degenerado para todo $t \in \mathbb{R}$. 


\section{Capítulo 1}

\section{Teoría básica para el análisis de operadores}

En este capítulo reunimos elementos previos (definiciones, ejemplos, teoremas, etc.) que consideramos básicos e importantes para el desarrollo de este trabajo. Estos temas pueden encontrarse en libros de análisis funcional o de física matemática, por ejemplo, [Arr2], [Do], [HS], [RS1], [We], [Ka] y [Yo].

\subsection{La transformada de Fourier}

Sea $\mathscr{S}\left(\mathbb{R}^{n}\right)$ el espacio de Schwarz definido por

$$
\mathscr{S}\left(\mathbb{R}^{n}\right)=\left\{f \in C^{\infty}\left(\mathbb{R}^{n}\right)\left|\sup _{x}\right| x^{\alpha}\left(D^{\beta} f\right)(x) \mid<\infty \text {, para cada } \alpha, \beta \in \mathbb{N}_{0}^{n}\right\} \text {. }
$$

Dado que el espacio de las funciones en $C^{\infty}\left(\mathbb{R}^{n}\right)$ con soporte compacto, $C_{0}^{\infty}\left(\mathbb{R}^{n}\right)$, es subconjunto de $\mathscr{S}\left(\mathbb{R}^{n}\right)$, entonces el espacio de Schwarz es denso en $L^{2}\left(\mathbb{R}^{n}\right)$.

Definición 1.1. Un espacio localmente convexo sobre $\mathbb{C}$ es un espacio vectorial complejo $X$ y una familia de seminormas $\left\{\rho_{i}\right\}_{i \in I}$ en $X$ tal que

$$
\rho_{i}(x)=0, \forall i \in I \text { implica que } x=0 .
$$

El espacio $\mathscr{S}\left(\mathbb{R}^{n}\right)$ es un espacio localmente convexo donde la familia de seminormas [Do] está dada por la expresión $(k \in \mathbb{N})$

$$
\|\| f\left\|_{k}=\sum_{|\alpha| \leq k}\right\|(1+|x|)^{k} D^{\alpha} f(x) \|_{\infty}
$$


Considerando que la topología en $\mathscr{S}\left(\mathbb{R}^{n}\right)$ está generada por una familia numerable de seminormas, la topología anterior es metrizable. Para este caso, $\mathscr{S}\left(\mathbb{R}^{n}\right)$ es un espacio métrico completo. Una de las tranformaciones más importantes sobre $L^{2}\left(\mathbb{R}^{n}\right)$ que usaremos en este trabajo es la transformada de Fourier. Ésta se define inicialmente en $\mathscr{S}\left(\mathbb{R}^{n}\right)$ y se extiende por continuidad a todo $L^{2}\left(\mathbb{R}^{n}\right)$.

Definición 1.2. El operador transformada de Fourier $\mathcal{F}$, aplica en la función $f \in \mathscr{S}\left(\mathbb{R}^{n}\right)$, como $\mathcal{F} f=\hat{f}$, donde

$$
\hat{f}(\xi)=(2 \pi)^{-n / 2} \int_{\mathbb{R}^{n}} e^{-i x \cdot \xi} f(x) d x, \quad \forall \xi \in \mathbb{R}^{n},
$$

donde $x \cdot \xi=\sum_{i=1}^{n} x_{i} \xi_{i}$ si $x=\left(x_{1}, \cdots, x_{n}\right)$ y $\xi=\left(\xi_{1}, \cdots, \xi_{n}\right)$.

La integral en (1.1) se toma respecto a la medida de Lebesgue. De hecho, en todo este trabajo la integración se tomará respecto a la medida de Lebesgue $d x$, a menos que se especifique algo diferente. Se tiene que $\hat{f} \in \mathscr{S}\left(\mathbb{R}^{n}\right)$. Se define la transformada inversa de Fourier $\mathcal{F}^{-1}$ como $\mathcal{F}^{-1} f=\check{f}$, para $f \in \mathscr{S}\left(\mathbb{R}^{n}\right)$, donde

$$
\check{f}(x)=(2 \pi)^{-n / 2} \int_{\mathbb{R}^{n}} e^{i x \cdot \xi} f(\xi) d \xi, \quad \forall x \in \mathbb{R}^{n} .
$$

Las integrales en las definiciones de $\hat{f}$ y $\breve{f}$ tienen sentido pues $f$ es una función continua y de rápido decrecimiento, por tanto, integrable. Las funciones $\hat{f}$ y $\check{f}$ se conocen como, la transformada de Fourier de $f$ y la transforma$d a$ inversa de Fourier de $f$, respectivamente. A continuación presentamos algunos resultados importantes sobre estos operadores. Las pruebas de las proposiciones en este caso se pueden leer en libros como [RS1], [RS2] y [Do].

Para $f \in \mathscr{S}\left(\mathbb{R}^{n}\right)$, se cumplen las siguientes igualdades:

$$
\left(D^{\alpha} f\right)^{\wedge}(p)=(i p)^{\alpha} \hat{f}(p) \quad \text { y } \quad\left(x^{\alpha} f(x)\right)^{\wedge}(p)=i^{|\alpha|} D^{\alpha} \hat{f}(p) .
$$

Se tiene asi, que el mapeo $\mathcal{F}: \mathscr{S}\left(\mathbb{R}^{n}\right) \longrightarrow \mathscr{S}\left(\mathbb{R}^{n}\right)$ es biyectivo y bicontinuo. De hecho, $\mathcal{F}^{-1}$ es precisamente el operador inverso de $\mathcal{F}$. Enunciamos en seguida una proposición que afirma que $\mathcal{F}$ es unitario en $\mathscr{S}\left(\mathbb{R}^{n}\right)$, fundamental en el desarrollo de nuestro trabajo.

Proposición 1.1 (Identidad de Parseval). Para $f$ y $g$ en $\mathscr{S}\left(\mathbb{R}^{n}\right)$, se cumple la igualdad

$$
(g, h)=(\hat{g}, \hat{h}) .
$$


En particular, si $\|\cdot\|_{2}$ denota la norma usual en $L^{2}\left(\mathbb{R}^{n}\right)$,

$$
\|f\|_{2}=\|\hat{f}\|_{2}, \forall f \in \mathscr{S}\left(\mathbb{R}^{n}\right) .
$$

Proposición 1.2 (Lema de Riemann-Lebesgue). La transformada de Fourier se extiende de manera única a un operador acotado de $L^{1}\left(\mathbb{R}^{n}, d x\right)$, las funciones Lebesgue-integrables, en $C_{\infty}\left(\mathbb{R}^{n}\right)$, las funciones continuas que se anulan en el infinito.

Definición 1.3. Un operador acotado $U$ sobre un espacio de Hilbert $\mathscr{H}$ con producto escalar $(\cdot, \cdot)$, es unitario si (a) $(U \phi, U \psi)=(\phi, \psi)$, para cualesquiera $\phi, \psi \in \mathscr{H}$ y, (b) $\operatorname{Ran} U=\mathscr{H}$.

Según el teorema de Plancherel, el operador transformada de Fourier se define sobre $L^{2}\left(\mathbb{R}^{n}\right)$ como la isometría obtenida al extender la transformada de Fourier en el espacio $\mathscr{S}\left(\mathbb{R}^{n}\right)$ a todo el espacio $L^{2}\left(\mathbb{R}^{n}\right)$. Esta extensión es única pues $\mathscr{S}\left(\mathbb{R}^{n}\right)$ es denso en $L^{2}\left(\mathbb{R}^{n}\right)$. El operador $\mathcal{F}: L^{2}\left(\mathbb{R}^{n}\right) \rightarrow L^{2}\left(\mathbb{R}^{n}\right)$ obtenido asi es unitario. Explícitamente, si $f \in L^{2}\left(\mathbb{R}^{n}\right), \hat{f}$ puede tomarse como

$$
\hat{f}(\xi)=\lim _{k \rightarrow \infty}(2 \pi)^{-n / 2} \int_{|x| \leq k} e^{-i x \cdot \xi} f(x) d x
$$

donde el límite se considera respecto a la topología inducida por la norma usual en $L^{2}\left(\mathbb{R}^{n}\right)$.

Ejemplo 1.1. Aplicamos la teoría anterior al operador de Laplace (Laplaciano) $\Delta$, operador diferencial de segundo orden, que en coordenadas cartesianas sobre $\mathbb{R}^{n}$ esta definido (formalmente) por

$$
\Delta:=\sum_{i=1}^{n} \frac{\partial^{2}}{\partial x_{i}^{2}}
$$

Este operador es de gran interés en Física Cuántica dado que se utiliza para definir el operador de Schrödinger de un sistema cuántico (ver [HS]). Para $f \in \mathscr{S}\left(\mathbb{R}^{n}\right)$, aplicando el teorema de la convergencia dominada de Lebesgue [AW, p. 126], se obtienen las siguientes propiedades para la transformada de Fourier

1. $\mathcal{F}\left(\partial f / \partial x_{k}\right)(\xi)=-i \xi_{k}(\mathcal{F} f)(\xi)$.

2. $\mathcal{F}\left(x_{k} f\right)(\xi)=i\left(\partial(\mathcal{F} f) / \partial \xi_{k}\right)(\xi)$. 
donde $x=\left\langle x_{1}, \cdots, x_{n}\right\rangle$ y $\xi=\left\langle\xi_{1}, \cdots, \xi_{n}\right\rangle$. Cuando aplicamos repetidamente estas propiedades, hallamos que

$$
\mathcal{F}(\Delta f)(x)=-x^{2}(\mathcal{F} f)(x),
$$

donde $x^{2}:=x_{1}^{2}+\cdots+x_{n^{2}}^{2}$. Puesto que $f(k)=(2 \pi)^{-n / 2} \int_{\mathbb{R}^{n}} e^{i k \cdot x} \hat{f}(x) d x$, el lado izquierdo de la igualdad anterior puede escribirse como

$$
\left(\mathcal{F} \Delta \mathcal{F}^{-1}\right) \hat{f}(x)=-x^{2} \hat{f}(x)
$$

ó

$$
\mathcal{F} \Delta \mathcal{F}^{-1}=-x^{2}
$$

Otro ejemplo más interesante es el siguiente

Ejemplo 1.2. Consideremos la ecuación de Klein-Gordon con potencial escalar,

$$
-\frac{\partial^{2}}{\partial t^{2}} \Psi(x, t)=(-\Delta-E \cdot x+q(x)) \Psi(x, t)
$$

con $x \in \mathbb{R}^{n}, t \in \mathbb{R}$. El vector $E \in \mathbb{R}^{n}$ es constante. Además, $\Delta$ es el Laplaciano y $q(x)-E \cdot x$ es un campo escalar. Consideremos el caso particular $E:=$ $\vec{e}_{1}=(1,0, \cdots, 0)$, de manera que (1.3) se reduce a

$$
-\frac{\partial^{2}}{\partial t^{2}} \Psi(x, t)=\left(-\Delta-x_{1}+q(x)\right) \Psi(x, t) .
$$

La ecuación (1.4) describe una partícula relativista con espín cero y masa cero en la presencia de un potencial escalar. Haciendo

$$
\bar{f}=\left(\begin{array}{c}
\psi(x, t) \\
i \frac{\partial}{\partial t} \psi(x, t)
\end{array}\right)
$$

(1.4) es equivalente a

$$
i \frac{\partial}{\partial t} \bar{f}=G \bar{f}
$$

con $G$ dado por

$$
G=\left(\begin{array}{ll}
0 & 1 \\
L & 0
\end{array}\right)
$$

donde

$$
L=-\Delta-x_{1}+q .
$$


Consideremos el caso en que $q \equiv 0$. Así, de (1.6), definimos $L_{0}=-\Delta-x_{1}$, operador que tomamos con dominio el espacio $\mathscr{S}\left(\mathbb{R}^{n}\right)$. Por el Ejemplo 1.1, $-\Delta=\mathcal{F}^{-1}\left(\xi_{1}^{2}+\xi_{\perp}^{2}\right) \mathcal{F}$, donde $\xi=\left\langle\xi_{1}, \xi_{\perp}\right\rangle=\left\langle\xi_{1}, \cdots, \xi_{n}\right\rangle$. Para $\psi \in \mathscr{S}\left(\mathbb{R}^{n}\right)$, al integrar por partes se obtiene

$$
\left(\mathcal{F}^{-1} i \frac{\partial}{\partial \xi_{1}} \mathcal{F} \psi\right)(x)=(2 \pi)^{-n / 2} \int_{\mathbb{R}^{n}} i e^{i x \cdot \xi} \frac{\partial}{\partial \xi_{1}}(\mathcal{F} \psi)(\xi) d \xi=x_{1} \psi(x)
$$

De manera similar,

$$
L_{0} \psi=e^{-i \xi_{1}^{3} / 3}\left(\xi_{\perp}^{2}-x_{1}\right) e^{i \xi_{1}^{3} / 3} \psi
$$

Observemos que

$$
\left[\left(\xi_{\perp}-x_{1}\right) \psi\right](x)=(2 \pi)^{-(n-1) / 2} \int_{\mathbb{R}^{n-1}} e^{i x_{\perp} \cdot y_{\perp}}\left(y_{\perp}^{2}-x_{1}\right)\left(\mathcal{F}_{\perp} \psi\right)\left(x_{1}, y_{\perp}\right) d y_{\perp}
$$

donde $\mathcal{F}_{\perp}$ denota la transformada de Fourier respecto a las variables $x_{\perp}=$ $\left\langle x_{2}, \cdots, x_{n}\right\rangle$. Puesto que $\mathcal{F}_{\perp}$ es un operador unitario y se tiene también que $e^{-i \xi_{1}^{3} / 3}$ es unitario, se concluye que $L_{0}$ es unitariamente equivalente al operador de multiplicación por la función $\xi_{\perp}^{2}-x_{1}$.

\subsection{Teorema Espectral y Teorema de Stone}

La teoría espectral se considerará sobre operadores autoadjuntos.

Definición 1.4. Sea $\mathscr{H}$ un espacio de Hilbert con producto escalar $(\cdot, \cdot)$. La gráfica de una transformación lineal $T: D(T) \longrightarrow \mathscr{H}$ con $D(T) \subset \mathscr{H}^{1}$, es el conjunto

$$
\Gamma(T)=\{\langle\phi, T \phi\rangle \mid \phi \in D(T)\} \subset \mathscr{H} \times \mathscr{H} .
$$

El espacio $\mathscr{H} \times \mathscr{H}$ es un espacio de Hilbert con producto escalar

$$
\left(\left\langle\phi_{1}, \psi_{1}\right\rangle,\left\langle\phi_{2}, \psi_{2}\right\rangle\right):=\left(\phi_{1}, \phi_{2}\right)+\left(\psi_{1}, \psi_{2}\right) .
$$

Decimos que el operador $T$ es cerrado cuando su gráfica, $\Gamma(T)$, es un subespacio cerrado de $\mathscr{H} \times \mathscr{H}$ respecto a la topología generada por la norma

$$
\|\langle\phi, \psi\rangle\|=\sqrt{\|\phi\|^{2}+\|\psi\|^{2}},
$$

misma que se obtiene de (1.7).

\footnotetext{
${ }^{1}$ Supondremos siempre que $D(T)$ es un subespacio denso en $\mathscr{H}$.
} 
Asociado a cada operador $A$ existe un único operador, llamado su adjunto, que es una herramienta poderosa en el estudio del espectro.

Definición 1.5. Sea $T$ un operador en un espacio de Hilbert $\mathscr{H}$ con producto escalar $(\cdot, \cdot)$ y dominio $D(T)$, un subespacio denso en $\mathscr{H}$.

1. $D\left(T^{*}\right)$ es el conjunto de vectores $\phi \in \mathscr{H}$ para los cuales existe un vector $\eta \in \mathscr{H}$ tal que

$$
(T \psi, \phi)=(\psi, \eta), \quad \text { para todo } \psi \in D(T) .
$$

En este caso, definimos $T^{*} \phi=\eta$. El operador $T^{*}$ se denomina el operador adjunto de $T$.

2. $T$ es simétrico si $T^{*}$ es una extensión de $T$, es decir, $D(T) \subset D\left(T^{*}\right)$ y $\left.T^{*}\right|_{D(T)}=T$.

3. $T$ se llama autoadjunto si $T=T^{*}$.

El siguiente teorema ofrece un criterio muy util para determinar cuándo un operador simétrico es autoadjunto.

Proposición 1.3 (Criterio para operadores autoadjuntos). Sea $T$ un operador simétrico en un espacio de Hilbert $\mathscr{H}$. $T$ es autoadjunto si y sólo si $T$ es cerrado y $\operatorname{Nuc}\left(T^{*} \mp i\right):=\left\{\psi \in D\left(T^{*}\right) \mid\left(T^{*} \mp i\right) \psi=0\right\}=\{0\}$, o bien, de manera equivalente, $T$ es cerrado y $\operatorname{Ran}(T \pm i):=\{(T \pm i) \psi \mid \psi \in D(T)\}=\mathscr{H}$.

Definición 1.6 (Espacio de Sobolev). Consideremos el espacio normado de las funciones $\phi \in C^{m}\left(\mathbb{R}^{n}\right)$ tal que $\|\phi\|_{p, m}<\infty$, donde

$$
\|\phi\|_{p, m}:=\left(\sum_{0 \leq|\alpha| \leq m}\left\|D^{\alpha} \phi\right\|_{p}^{p}\right)^{1 / p}
$$

Aquí, $\|\phi\|_{p}$ es la norma en el espacio $L^{p}\left(\mathbb{R}^{n}, d x\right)$, para $1 \leq p<\infty$. Se define el espacio de Sobolev de orden $m, H^{p, m}\left(\mathbb{R}^{n}\right)$, como la completitud de $\left(C^{m}\left(\mathbb{R}^{n}\right),\|\cdot\|_{p, m}\right)$. Si $p=2$, se denota el espacio de Sobolev de orden $m$ por $H^{m}\left(\mathbb{R}^{n}\right)$. El espacio $H^{m}\left(\mathbb{R}^{n}\right)$ es un espacio de Hilbert [HS].

Definición 1.7. Sean $\langle M, \mu\rangle$ un espacio de medida y $f: M \longrightarrow \mathbb{R}$ una función medible. Decimos que $x \in \mathbb{R}$ pertenece al rango esencial de $f$, si $y$ sólo si, para todo $\epsilon>0$,

$$
0<\mu(\{m \in M|| f(m)-x \mid<\epsilon\}) .
$$


En el caso en que $f$ es continua, el rango esencial de $f$ es igual a la cerradura de su rango.

Definición 1.8 (Espectro y espectro esencial de un operador). Sea $A$ un operador cerrado en un espacio de Hilbert $\mathscr{H}$, con dominio $D(A)$,

1. Decimos que un número complejo $\lambda$ pertenece al conjunto resolvente de $A, \rho(A)$, si el operador $(A-\lambda I)^{-1}$ existe y es acotado ( $I$ es el operador identidad). El espectro de $A, \sigma(A)$, se define como $\sigma(A)=\mathbb{C} \backslash \rho(A)$.

2. Un vector $u$ no nulo que satisface $A u=\lambda u$, con $\lambda \in \mathbb{C}$, se denomina vector propio y $\lambda$ es el valor propio correspondiente.

3. Si $\lambda$ es un punto aislado de $\sigma(A)$ y es un valor propio de multiplicidad finita $^{2}$, entonces decimos que $\lambda \in \sigma_{d}(A)$, el espectro discreto de $A$. El espectro esencial de $A, \sigma_{\text {ess }}(A)$, se define como $\sigma_{\text {ess }}(A)=\sigma(A) \backslash \sigma_{d}(A)$.

Las transformaciones isométricas (mapeos que preservan norma) son muy útiles en mecánica cuántica. Cuando $U$ es unitario, es decir, es un operador isométrico, acotado y sobreyectivo, y, $A$ un operador cerrado, la conjugación del operador $A$ por $U$, definido por

$$
A_{U}:=U A U^{-1},
$$

con $D\left(A_{U}\right)=U(D(A)$, es útil en el análisis espectral de $A$, tal como lo muestra el siguiente teorema.

Proposición 1.4. Sea $A$ un operador cerrado y $U$ un operador unitario. Entonces se cumplen:

1. $A_{U}$ es cerrado sobre $D\left(A_{U}\right)$ y $\sigma\left(A_{U}\right)=\sigma(A)$;

2. Si $A$ es autoadjunto, $A_{U}$ es autoadjunto.

Proposición 1.5. Consideremos un espacio de medida finita $\langle M, \mu\rangle$. Si $f$ : $M \longrightarrow \mathbb{R}$ es una función medible $\mathrm{y}$, finita casi en todas partes, entonces el operador $T_{f}(\varphi):=f \varphi$ sobre $L^{2}(M, d \mu)$, con dominio

$$
D\left(T_{f}\right)=\left\{\varphi \mid f \varphi \in L^{2}(M, d \mu)\right\},
$$

es autoadjunto y su espectro $\sigma\left(T_{f}\right)$ coincide con el rango esencial de $f$.

\footnotetext{
${ }^{2}$ Esto significa que el subespacio $\{\phi \in \mathscr{H} \mid A \phi=\lambda \phi\}$ es de dimensión finita.
} 
Teorema 1.1 (Teorema Espectral en su forma de operador de multiplicación). Sea $A$ un operador autoadjunto sobre un espacio de Hilbert separable $\mathscr{H}$ con dominio $D(A)$. Entonces existen un espacio de medida $\langle M, \mu\rangle$, con medida finita $\mu$, un operador unitario $U: \mathscr{H} \rightarrow L^{2}(M, d \mu)$, y una función $f: M \longrightarrow \overline{\mathbb{R}}=\mathbb{R} \cup\{ \pm \infty\}$, finita casi en todas partes, tales que,

(a) $\phi \in D(A)$ si y solo si $f(\cdot)(U \phi)(\cdot) \in L^{2}(M, d \mu)$.

(b) Si $\varphi \in U[D(A)]$, entonces $\left(U A U^{-1} \varphi\right)(m)=f(m) \varphi(m)$.

Ejemplo 1.3. Aplicaremos el resultado anterior a nuestros dos ejemplos. Considerando que la medida de Lebesgue no es finita, podemos construir un mapeo unitario del espacio de medida $\sigma$-finita $\left(\mathbb{R}^{n}, d x\right)$ a un espacio de medida finita $\left(\mathbb{R}^{n}, \mu\right)$ finita de la siguiente manera: Sea $h \in L^{1}\left(\mathbb{R}^{n}, d x\right)$ con $h>0$. Hagamos $d \mu:=h d x$. Consideremos el mapeo $V: L^{2}\left(\mathbb{R}^{n}, d x\right) \longrightarrow L^{2}\left(\mathbb{R}^{n}, d \mu\right)$, donde

$$
V f=\frac{f}{\sqrt{h}} .
$$

Observemos que

$$
\begin{aligned}
\|V f\|_{L^{2}\left(\mathbb{R}^{n}, d \mu\right)} & =\int_{\mathbb{R}^{n}} \frac{|f|^{2}}{h} d \mu \\
& =\int_{\mathbb{R}^{n}}|f|^{2} d x \\
& =\|V f\|_{L^{2}\left(\mathbb{R}^{n}, d x\right)} .
\end{aligned}
$$

Así, en los ejemplos 1.1 y 1.2 que hemos estado considerando, el operador unitario $V$ al que se refiere el teorema anterior es la composición de la transformada de Fourier con el operador $V$.

Ejemplo 1.4. Considerando que los operadores de los ejemplos 1.1 y 1.2 son unitariamente equivalentes a los operadores de multiplicación por $f(x)=-x^{2}$ y por $f(x)=\left(x_{2}^{2}+\cdots+x_{n}^{2}\right)-x_{1}$, respectivamente, se deduce, según el teorema anterior, que los operadores $-\Delta$ y $L_{0}$ son autoadjuntos. Además, $\sigma(-\Delta)=$ $[0,+\infty)$ y $\sigma\left(L_{0}\right)=\mathbb{R}$, pues las funciones $f(x)=x^{2}$ y $f(x)=\left(x_{2}^{2}+\cdots+x_{n}^{2}\right)-x_{1}$ son continuas y sus imágenes son $[0, \infty)$ y $\mathbb{R}$, respectivamente.

En relación con el teorema 1.1, para cualquier operador autoadjunto $A$, dada una función de Borel acotada $h$ sobre $\mathbb{R}$, se define

$$
h(A):=U^{-1} T_{h(f)} U,
$$


donde $T_{h(f)}$ es el operador sobre $L^{2}(M, d \mu)$ el cual actúa por multiplicación por la función $h \circ f$. De esta observación y del teorema 1.1, se sigue otra versión del teorema espectral. Denotemos por

$$
\mathscr{L}(\mathscr{H}):=\{T: \mathscr{H} \longrightarrow \mathscr{H} \mid T \text { es operador lineal continuo }\} .
$$

Teorema 1.2 (Teorema espectral en su forma de cálculo funcional). Sea $A$ un operador autoadjunto sobre un espacio de Hilbert separable $\mathscr{H}$. Entonces existe un único mapeo $\phi: \mathbb{B}_{a}(\mathbb{R}) \longrightarrow \mathscr{L}(\mathscr{H})$, donde $\mathbb{B}_{a}(\mathbb{R})$ representa el conjunto de las funciones complejas de Borel acotadas, y cumpliendo

(a) $\phi$ es un *-homomorfismo algebraico, es decir, $\phi$ cumple

$$
\begin{aligned}
& \text { - } \phi(f g)=\phi(f) \phi(g) . \\
& \text { - } \phi(\lambda f)=\lambda \phi(f) . \\
& \text { - } \phi(1)=I . \\
& \text { - } \phi(\bar{f})=\phi(f)^{*} .
\end{aligned}
$$

(b) $\phi$ es continuo, esto es, $\|\phi(h)\|_{\mathscr{S C}(\mathscr{C})} \leq\|h\|_{\infty}$, para cada $h \in \mathbb{B}_{a}(\mathbb{R})$.

(c) Sea $\left\{h_{n}(x): n \in \mathbb{N}\right\} \subset \mathbb{B}_{a}(\mathbb{R})$ con $h_{n}(x) \rightarrow x$ para cada $x$ y $\left|h_{n}(x)\right| \leq|x|$ para toda $x$ y toda $n$. Entonces, para cualquier $\psi \in D(A)$,

$$
\lim _{n \rightarrow \infty} \phi\left(h_{n}\right) \psi=A \psi
$$

(d) Si $h_{n}(x) \rightarrow h(x)$ para toda $x$ y si la sucesión $\left\{\left\|h_{n}\right\|_{\infty}\right\}$ es acotada, entonces $\left\|\phi\left(h_{n}\right)-\phi(h)\right\|_{\mathscr{L}(\mathscr{H})} \rightarrow 0$.

(e) Si $A \psi=\lambda \psi$ entonces $\phi(h) \psi=h(\lambda) \psi$.

(f) Si $h \geq 0$ entonces $\phi(h) \geq 0$.

El teorema espectral en su forma de medida valuada sobre proyecciones. se sigue del cálculo funcional. Sea $P_{\Omega}$ el operador $\chi_{\Omega}(A)$, donde $\chi_{\Omega}$ es la función característica del conjunto Borel medible $\Omega \subset \mathbb{R}$. Para cada $\phi \in \mathscr{H}$, $\Omega \mapsto\left(\phi, P_{\Omega} \phi\right)$ es una medida de Borel sobre $\mathbb{R}$ que se denota por $d\left(\phi, P_{\lambda} \phi\right)$. La medida compleja $d\left(\phi, P_{\lambda} \phi\right)$ esta definida por la identidad de polarización. Ahora, dada una función de Borel acotada $g$, definimos $g(A)$ por

$$
(\phi, g(A) \phi)=\int_{\mathbf{R}} g(\lambda) d\left(\phi, P_{\lambda} \phi\right)
$$


donde $g(A)$ coincide con la definición dada anteriormente en el teorema (1.1), es decir, $g(A)=U^{-1} T_{g(f)} U$. Cuando la función $g$ de Borel no es acotada, se define

$$
D_{g}=\left\{\left.\phi \in \mathscr{H}\left|\int_{\mathbf{R}}\right| g(\lambda)\right|^{2} d\left(\phi, P_{\lambda} \phi\right)<\infty\right\} .
$$

Asi, $D_{g}$ es denso en $\mathscr{H}$ y el operador $g(A)$ sobre $D_{g}$ se define como

$$
(\phi, g(A) \phi)=\int_{\mathbb{R}} g(\lambda) d\left(\phi, P_{\lambda} \phi\right) .
$$

Denotamos $g(A)$ definida previamente como

$$
g(A):=\int_{\mathbf{R}} g(\lambda) d P_{\lambda} .
$$

En particular, para $\phi, \psi \in D(A)$,

$$
(\phi, A \psi)=\int_{\mathbb{R}} \lambda d\left(\phi, P_{\lambda} \psi\right)
$$

El siguiente teorema resume lo anterior.

Teorema 1.3 (Teorema espectral en su forma de medida valuada sobre proyecciones). Existe una correspondencia uno a uno entre operadores autoadjuntos $A$ y medidas valuadas sobre proyecciones $\left\{P_{\Omega}\right\}$ sobre $\mathscr{H}$, dada por la correspondencia

$$
A=\int_{\mathbf{R}} \lambda d P_{\lambda} .
$$

Si $g$ es una función a valores reales de Borel sobre $\mathbb{R}$, entonces

$$
g(A)=\int_{\mathbf{R}} g(\lambda) d P_{\lambda}
$$

definida sobre $D_{g}$, es autoadjunto.

El siguiente teorema usa el cálculo funcional para definir $e^{i t A}$, en un espacio de Hilbert, cuando $A$ es autoadjunto.

Teorema 1.4. Sea $A$ un operador autoadjunto y definimos (usando (1.9)) $U(t)=e^{i t A}$. Entonces 
(a) Para cada $t \in \mathbb{R}, U(t)$ es un operador unitario y $U(t+s)=U(t) U(s)$ para cualesquiera $t, s \in \mathbb{R}$.

(b) Si $\varphi \in \mathscr{H}$ y $t \rightarrow t_{0}$, entonces $U(t) \varphi \longrightarrow U\left(t_{0}\right) \varphi$, en la norma de $\mathscr{H}$.

(c) Para $\psi \in D(A), \frac{U(t) \psi-\psi}{t} \longrightarrow i A \psi$ cuando $t \rightarrow 0$, en la norma de $\mathscr{H}$.

(d) Si $\lim _{t \rightarrow 0} \frac{U(t) \psi-\psi}{t}$ existe en la norma de $\mathscr{H}$, entonces $\psi \in D(A)$.

Teorema 1.5 (Teorema de Stone). Sea $U(t)$ cumpliendo los incisos (a) y (b) del teorema anterior, sobre un espacio de Hilbert $\mathscr{H}$. Entonces, existe un único operador autoadjunto $A$ sobre $\mathscr{H}$ tal que $U(t)=e^{i t A}$.

\subsection{Espectro esencial y teorema de Weyl}

El teorema de Weyl garantiza la estabilidad del espectro esencial de operadores autoadjuntos bajo ciertas perturbaciones llamadas perturbaciones relativamente compactas. Consideremos operadores autoadjuntos sobre un espacio de Hilbert $\mathscr{H}$.

Definamos antes la norma de un operador. Sean $\mathscr{H}_{1}$ y $\mathscr{H}_{2}$ espacios normados. Un operador $A: D(A) \subset \mathscr{H}_{1} \rightarrow \mathscr{H}_{2}$ se dice acotado si existe $C \geq 0$ tal que $\|T \phi\| \leq C\|\phi\|$ para todo $\phi \in D(A)$. Para un operador acotado $A$ de $\mathscr{H}_{1}$ en $\mathscr{K}_{2}$ se define la norma $\|A\|$ por

$$
\|A\|=\inf \{C \geq 0:\|T \phi\| \leq C\|\phi\| \text { para todo } \phi \in D(A)\} .
$$

Ahora recordamos la definición de un operador compacto. Un conjunto se dice precompacto cuando su clausura es compacto. Sean $X$ e $Y$ espacios de Banach. Un operador $T \in \mathscr{L}(X, Y)$ se llama compacto si manda conjuntos acotados de $X$ en conjuntos precompactos de $Y$. Equivalentemente, $T$ es compacto si y sólo si para cada sucesión acotada $\left\{x_{n}\right\} \subset X,\left\{T x_{n}\right\}$ tiene una subsucesión convergente en $Y$. Pero en espacios de Hilbert, ésta es equivalente a la siguiente [AJS].

Definición 1.9. Un operador $T$ de la forma

$$
T \varphi=\sum_{i=1}^{m}\left(\phi_{i}, \varphi\right) \psi_{i}, \quad \forall \varphi \in \mathscr{H}
$$


donde $\left\{\phi_{i}, \psi_{i}\right\} \subset \mathscr{H}$ y $m$ es finito, se llama operador de rango finito. Un operador acotado $A$ se llama compacto si existe una sucesión de operadores de rango finito $\left\{T_{m}\right\}_{m \in \mathbb{N}}$ tal que

$$
\lim _{m \rightarrow \infty}\left\|T_{m}-A\right\|=0 .
$$

Definición 1.10. Sea $A$ un operador cerrado con $\rho(A) \neq \emptyset$. Un operador $B$ es llamado $A$-compacto si,

1. $D(A) \subset D(B)$;

2. $B R_{A}(z)$ es compacto para algún $z \in \rho(A)$, donde $R_{A}(z):=(A-z I)^{-1}$.

Enunciamos el resultado sobre la invariancia del espectro esencial que hemos mencionado.

Teorema 1.6 (Teorema de Weyl). Sean $A$ un operador autoadjunto y $B$ un operador simétrico. Si $B$ es $A$-compacto. Entonces,

$$
\sigma_{\text {ess }}(A+B)=\sigma_{\text {ess }}(A) .
$$

Un resultado importante que usaremos (combinado con el teorema de Weyl) en los capítulos posteriores es el siguiente.

Teorema 1.7. [HS, p.73] El espectro del operador autoadjunto $-\Delta$ sobre $H^{2}\left(\mathbb{R}^{n}\right)$ es $\sigma(-\Delta)=\sigma_{\text {ess }}(-\Delta)=[0, \infty)$.

\subsection{Convergencia de operadores no acotados}

En esta sección, definimos ciertos tipos de convergencia de operadores y enunciamos algunos teoremas al respecto.

Definición 1.11. Sea $\mathcal{A}=\left\{A_{n} \mid n \in \mathbb{N}\right\}$ una familia numerable de operadores autoadjuntos. Decimos que $A_{n}$ converge al operador $A$ en el sentido de la resolvente en norma si $R_{\lambda}\left(A_{n}\right) \rightarrow R_{\lambda}(A)$ en norma, para cada $\lambda \in \mathbb{C} \backslash \mathbb{R}$. Decimos que $A_{n}$ converge al operador $A$ en el sentido de la resolvente fuertemente si $R_{\lambda}\left(A_{n}\right) \rightarrow R_{\lambda}(A)$ fuertemente para cada $\lambda \in \mathbb{C} \backslash \mathbb{R}$.

Teorema 1.8. Sean $A_{n}$ y $A$ operadoı es autoadjuntos. 
1. Si $A_{n} \rightarrow A$ en el sentido de la resolvente en norma y $f$ es una función continua sobre $\mathbb{R}$ que se anula en $+\infty$, entonces

$$
\left\|f\left(A_{n}\right)-f(A)\right\| \rightarrow 0 .
$$

2. Si $A_{n} \rightarrow A$ en el sentido de la resolvente fuertemente y $f$ es una función continua y acotada sobre $\mathbb{R}$, entonces

$$
f\left(A_{n}\right) \varphi \rightarrow f(A) \varphi, \quad \forall \varphi \in \mathscr{H} .
$$

Un resultado que usaremos frecuentemente en el trabajo esta dado por el siguiente teorema.

Teorema 1.9. Sea $P_{A}(t)$ un operador continuo en la norma de operadores para cada $t \in \mathbb{R}$. Si $P_{A}^{2}(t)=P_{A}(t)$ y $P_{A}^{*}(t)=P_{A}(t), \forall t \in \mathbb{R}$, entonces $\operatorname{dim} P_{A}(t)$ es constante.

Demostración. Sea $t_{0} \in \mathbb{R}$. Sin pérdida de generalidad, podemos considerar que $\operatorname{dim} P_{A}\left(t_{0}\right)=n$, para algún $n \in \mathbb{N}$.

Consideremos $0<\epsilon<1$. En este caso, existe $\delta>0$ tal que

$$
\left|t-t_{0}\right|<\delta \Rightarrow\left\|P_{A}(t)-P_{A}\left(t_{0}\right)\right\|<\frac{\epsilon}{\sqrt{n}} .
$$

Supongamos que $\left\{\varphi_{1}\left(t_{0}\right), \ldots, \varphi_{n}\left(t_{0}\right)\right\}$ es una base ortonormal de $\operatorname{Ran} P_{A}\left(t_{0}\right)$. Entonces, si para alguna combinación lineal de esta base se cumple

$$
\left\|\sum_{i=1}^{n} c_{i} P_{A}(t) \varphi_{i}\left(t_{0}\right)\right\|=0
$$

entonces, puesto que $P_{A}\left(t_{0}\right) \varphi_{i}\left(t_{0}\right)=\varphi_{i}\left(t_{0}\right), \quad \forall i=1, \ldots, n$,

$$
\begin{aligned}
\left\|\sum_{i=1}^{n} c_{i} P_{A}\left(t_{0}\right) \varphi_{i}\left(t_{0}\right)\right\| & =\left(\sum_{i=1}^{n}\left|c_{i}\right|^{2}\right)^{1 / 2} \\
& \leq\left\|\sum_{i=1}^{n} c_{i}\left(P_{A}(t)-P_{A}\left(t_{0}\right)\right) \varphi_{i}\left(t_{0}\right)\right\| \\
& \leq \sum_{i=1}^{n}\left|c_{i}\right|\left\|\mid\left(P_{A}(t)-P_{A}\left(t_{0}\right)\right)\right\| \\
& \leq\left(\sum_{i=1}^{n}\left|c_{i}\right|^{2}\right)^{1 / 2} \epsilon .
\end{aligned}
$$


lo cual es una contradicción. Por tanto, $\operatorname{Ran} P_{A}\left(t_{0}\right) \leq \operatorname{Ran} P_{A}(t)$. Intercambiando $t$ por $t_{0}$ obtenemos la otra desigualdad. 


\section{Capítulo 2}

\section{Estudio del espectro discreto de un hamiltoniano dependiente del tiempo asociado al modelo del parámetro de impacto}

\subsection{Introducción}

Consideramos un proceso donde para cada tiempo $t$ la energía del sistema está representada por el operador $H(t)$. Si $\phi$ es el estado inicial del sistema para $t=-\infty$ con energía asociada $E_{i}$, y si se sabe que durante el proceso la variación de la energía $\nu(H(t)):=E_{t}-E_{i}$ satisface para algún $\epsilon>0$,

$$
-\epsilon<\nu(H(t))<\epsilon,
$$

donde $E_{t}$ es la energía asociada a $H(t)$, entonces los estados energéticos probables durante el proceso corresponderán a los estados del sistema con energía asociada $E_{t}$ en el intervalo $\left(E_{i}-\epsilon, E_{i}+\epsilon\right)$. Esos estados se aproximan asintóticamente al estado final $\tilde{\phi}$ (es decir, cuando $t=+\infty$ ). La aproximación nos permite dar una estimación de la probabilidad de transición entre el estado inicial y el final. Por esta razón, se necesita tener un conocimiento preciso del espectro discreto del operador $H(t)$. Asi, nos proponemos en este capítulo estudiar el espectro de un operador dependiente del tiempo asociado al modelo del parámtero de impacto, y caracterizar los valores propios correspondientes al espectro discreto. 


\subsection{Definición del Hamiltoniano}

En [Arr1] se considera para todo $t \in \mathbb{R}$ el operador

$$
H_{v}(t)=-\frac{1}{2} \Delta-\lambda_{1} V-\lambda_{2} V_{\rho}
$$

con dominio $D\left(H_{v}(t)\right)=H^{2}\left(\mathbb{R}^{n}\right)$. Los números reales $\lambda_{1}, \lambda_{2}$, cumplen $\lambda_{1}>$ $\lambda_{2}>0$. El operador $V$ se define por

$$
V \varphi=(g, \varphi) g, \quad \forall \varphi \in L^{2}\left(\mathbb{R}^{n}\right),
$$

donde $g$ es un vector fijo no nulo en $L^{2}\left(\mathbb{R}^{n}\right)$ y $(\cdot, \cdot)$ denota el producto escalar usual en el espacio de Hilbert $L^{2}\left(\mathbb{R}^{n}\right)$. El operador $V_{\rho}$ se define como

$$
V_{\rho} \varphi=\left(g_{\rho}, \varphi\right) g_{\rho}, \quad \forall \varphi \in L^{2}\left(\mathbb{R}^{n}\right)
$$

donde

$$
g_{\rho}(x)=g(x-\rho(t)) .
$$

con $\rho$ una función continua de $\mathbb{R}$ a $\mathbb{R}^{n}$, dos veces diferenciable que cumple, para algún $t_{ \pm}>0$,

$$
\begin{gathered}
\left|\rho(t)-\overrightarrow{\mathbf{c}}_{ \pm}-\overrightarrow{\mathbf{v}}_{ \pm} t\right| \leq \frac{C_{0}}{|t|^{\alpha}}, \quad \forall \pm t>t_{ \pm} ; \\
\left|\frac{d^{k}}{d t^{k}}\left(\rho(t)-\overrightarrow{\mathbf{c}}_{ \pm}-\overrightarrow{\mathbf{v}}_{ \pm} t\right)\right| \leq C_{k} v^{k}, \quad \forall \pm t>t_{ \pm} \quad \text { y para } k=1,2 .
\end{gathered}
$$

donde $\alpha \in \mathbb{R}^{+}, \overrightarrow{\mathbf{c}}, \overrightarrow{\mathbf{v}} \in \mathbb{R}^{n}, \overrightarrow{\mathbf{v}} \neq \overrightarrow{\mathbf{0}}, v:=|\overrightarrow{\mathbf{v}}| \leq 1 \mathrm{y}$

$$
\left|\frac{d^{k}}{d t^{k}} \rho(t)\right| \leq v^{k}, \quad-t_{-} \leq t \leq t_{+} \quad \text { para } k=1,2
$$

Llamamos $M$ a la constante definida por

$$
M:=\operatorname{máx}\left\{t_{ \pm},\left|c_{ \pm}\right|, C_{0}, C_{1}, C_{2}\right\} .
$$

Así, $\rho$ se comporta como la recta $\overrightarrow{\mathbf{c}}_{ \pm}+\overrightarrow{\mathbf{v}}_{ \pm} t$ para $t$ suficientemente grande. 


\subsection{Interpretación física}

El operador $H_{v}(t)$ está asociado al modelo del parámetro de impacto para la dispersión de una partícula ligera y dos partículas pesadas. En este modelo se asume que las partículas pesadas se mueven a lo largo de trayectorias clásicas y que no son afectadas por la presencia de la partícula ligera. Se supone también que las partículas pesadas tienen una masa muy grande y su influencia sobre la partícula ligera esta representada por potenciales que dependen del tiempo. Consideraremos que los potenciales son separables. El vector propio correspondiente al ínfimo del espectro de $H_{v}(t)$ es llamado el estado fundamental del sistema $H_{v}(t)$.

\subsection{Espectros de operadores asociados}

Primero, estudiaremos el espectro de operadores menos complejos que nos permitirán demostrar ciertas propiedades de $H_{v}(t)$ en lo sucesivo. Consideraremos los operadores

$$
H_{i}:=-\frac{1}{2} \Delta-\lambda_{i} V, \quad i=1,2 .
$$

con $V$ defininido como en (2.2), con dominio $H^{2}\left(\mathbb{R}^{n}\right)$. Es bien conocido que el operador Laplaciano,

$$
H_{0}:=-\frac{1}{2} \Delta=-\frac{1}{2} \sum_{i=1}^{n} \frac{\partial^{2}}{\partial x_{i}^{2}}
$$

con dominio $D\left(H_{0}\right)=H^{2}\left(\mathbb{R}^{n}\right)$, es autoadjunto [HS] y su espectro $\sigma\left(H_{0}\right)$ coincide con su espectro esencial $\sigma_{\text {ess }}\left(H_{0}\right)=[0, \infty)$.

Usando el teorema de Weyl para $V$, el cual es compacto por tener rango de dimensión finita, se tiene para $i=1,2$,

$$
\sigma_{\text {ess }}\left(H_{i}\right)=\sigma_{e s s}\left(H_{0}\right)=[0, \infty) .
$$

Interesa saber ahora cuál es el espectro discreto de $H_{i}, i=1,2$. La siguiente proposición será de vital importancia en éste trabajo.

Proposición 2.1. El operador $\mathrm{H}_{2}$ tiene a lo más un valor propio negativo, $-Q_{2}$, y es de multiplicidad uno. 
Demostración. $\mathrm{Si}-Q$ es un valor propio negativo, existe $\overrightarrow{0} \neq \varphi \in D\left(H_{2}\right)$ tal que

$$
H_{2} \varphi=-Q \varphi
$$

Sustituyendo $\mathrm{H}_{2}$ y aplicando transformada de Fourier en ambos lados, obtenemos

$$
\frac{1}{2} p^{2} \hat{\varphi}(p)-\lambda_{2}(g, \varphi) \hat{g}(p)=-Q \hat{\varphi}(p)
$$

donde $p^{2}:=p_{1}^{2}+\cdots+p_{n}^{2}, p=\left(p_{1}, \cdots, p_{n}\right) \in \mathbb{R}^{n}$. Aqui usamos el hecho de que la transformada de Fourier de $-\frac{1}{2} \Delta(\varphi)$ es $\frac{1}{2} p^{2} \hat{\varphi}$. Lo anterior implica

$$
\hat{\varphi}(p)=\lambda_{2}(g, \varphi) \frac{\hat{g}(p)}{\frac{1}{2} p^{2}+Q}
$$

Entonces

$$
\begin{aligned}
(\hat{g}, \hat{\varphi}) & =\lambda_{2}(g, \varphi)\left(\hat{g}, \frac{\hat{g}}{\frac{1}{2} p^{2}+Q}\right) \\
& =\lambda_{2}(g, \varphi)\left(\frac{\hat{g}}{\left(\frac{1}{2} p^{2}+Q\right)^{1 / 2}}, \frac{\hat{g}}{\left(\frac{1}{2} p^{2}+Q\right)^{1 / 2}}\right) \\
& =\lambda_{2}(g, \varphi)\left\|\frac{\hat{g}}{\left(\frac{1}{2} p^{2}+Q\right)^{1 / 2}}\right\|^{2}
\end{aligned}
$$

Obtenemos así,

$$
\frac{1}{\lambda_{2}}=\left\|\frac{\hat{g}}{\left(\frac{1}{2} p^{2}+Q\right)^{1 / 2}}\right\|^{2} .
$$

Consideremos la función $\alpha: \mathbb{R}^{+} \longrightarrow \mathbb{R}$ dependiente de $Q$, definida por

$$
\alpha(Q):=\left\|\frac{\hat{g}}{\left(\frac{1}{2} p^{2}+Q\right)^{1 / 2}}\right\|^{2} .
$$

Derivando $\alpha$ respecto a $Q$ y usando el teorema de la convergencia dominada de Lebesgue, se obtiene

$$
\alpha^{\prime}(Q)=-\left\|\left(\frac{1}{2} p^{2}+Q\right)^{-1} \hat{g}\right\| .
$$


Por tanto, $\alpha$ es monótona decreciente y $\operatorname{Ran}(\alpha)=(0, I)$, con

$$
I=2 \int_{\mathbb{R}^{n}}\left|\frac{\hat{g}(p)}{p}\right|^{2} d p
$$

donde $I$ puede ser $+\infty$. De esta manera, para $\frac{1}{\lambda_{2}} \in(0, I)$, existe un único $Q_{2} \in \mathbb{R}^{+}$tal que $\alpha\left(Q_{2}\right)=\frac{1}{\lambda_{2}}$. En este caso, $-Q_{2}$ es valor propio único de $H_{2}$ y el vector propio correspondiente $\varphi$ cumple

$$
\hat{\varphi}=\frac{\hat{g}}{\frac{1}{2} p^{2}+Q_{2}} .
$$

En efecto, si $\alpha\left(Q_{2}\right)=\frac{1}{\lambda_{2}}$, entonces

$$
\begin{aligned}
\widehat{H_{2} \varphi} & =\frac{1}{2} p^{2} \frac{\hat{g}}{\frac{1}{2} p^{2}+Q_{2}}-\lambda_{2}\left(\hat{g}, \frac{\hat{g}}{\frac{1}{2} p^{2}+Q_{2}}\right) \hat{g} \\
& =\frac{1}{2} p^{2} \frac{\hat{g}}{\frac{1}{2} p^{2}+Q_{2}}-\lambda_{2}\left\|\frac{\hat{g}}{\left(\frac{1}{2} p^{2}+Q_{2}\right)^{1 / 2}}\right\|^{2} \hat{g} \\
& =\frac{1}{2} p^{2} \frac{\hat{g}}{\frac{1}{2} p^{2}+Q_{2}}-\hat{g} \\
& =-Q_{2} \hat{\varphi}
\end{aligned}
$$

Entonces $H_{2} \varphi=-Q_{2} \varphi$, pues la transformada es inyectiva en $D\left(H_{2}\right)$ [RS1]. De (2.12) vemos que la multiplicidad de $-Q_{2}$ es 1 .

Ahora usamos la demostración anterior para probar el siguiente corolario que se refiere al espectro discreto de operadores definidos de manera similar.

Corolario 2.1. Si $H_{2}$ tiene un único valor propio $-Q_{2}$, entonces los operadores $H_{1}$ y $H=-\frac{1}{2} \Delta-\left(\lambda_{1}+\lambda_{2}\right) V$ tambien tienen un único valor propio de multiplicidad $1,-Q_{1} \mathrm{y}-Q_{0}$, respectivamente. Además,

$$
-Q_{0}<-Q_{1}<-Q_{2} \text {. }
$$

Demostración. Como $\lambda_{1}+\lambda_{2}>\lambda_{1}>\lambda_{2}>0$, entonces

$$
\frac{1}{\lambda_{1}+\lambda_{2}}<\frac{1}{\lambda_{1}}<\frac{1}{\lambda_{2}}
$$

Dado que la función $\alpha$, definida en (2.13), es monótona decreciente, la existencia de $-Q_{2}$, valor propio de $H_{2}$, implica la existencia de $-Q_{1}$ y $-Q_{0}$, valores propios de $H_{1}$ y $H_{0}$, de multiplicidad 1 , respectivamente. Además, $Q_{2}<Q_{1}<Q_{0}$. 


\subsection{Caracterización del espectro discreto del hamiltoniano}

En esta sección, enunciamos y demostramos un resultado que caracteriza el espectro discreto de $H_{v}(t)$.

Teorema 2.1. Sean $g$ y $\rho$ como en (2.1)-(2.4) y

$$
-E(t)=\inf \left\{a \in \mathbb{R} \mid a \in \sigma\left(H_{\nu}(t)\right)\right\} .
$$

Supongamos que $H_{2}$ tiene valor propio $-Q_{2}$. Si $\lambda_{1}>\lambda_{2}>0$, entonces $-E(t)$ es de multiplicidad 1 y

$$
-E(t) \in\left[-E_{0},-Q_{1}\right], \quad \forall t \in \mathbb{R},
$$

donde $-E_{0}$ es el ínfimo del espectro del operador autoadjunto

$$
-\frac{1}{2} \Delta-\left(\lambda_{1}+\lambda_{2}\right) V
$$

y $-Q_{1}$ es el ínfimo del espectro de $H_{1}$.

Además, si existe otro valor propio negativo de $H_{v}(t)$, éste se halla en $\left[-Q_{2}, 0\right)$. Tambien, el vector propio para $-E(t)$ puede ser tomado como

$$
\Psi(t)=\mathcal{F}^{-1} \mathcal{Q}_{t}^{-1}\left(p^{2} / 2+E(t)\right)^{-1}\left(c(t) \hat{g}_{\rho}+\hat{g}\right)
$$

con

$$
c(t)=\frac{\lambda_{2}\left(\hat{g}_{\rho},\left(p^{2} / 2+E(t)\right)^{-1} \hat{g}\right)}{1-\lambda_{2}\left\|\left(p^{2} / 2+E(t)\right)^{-1 / 2} \hat{g}\right\|^{2}} .
$$

y $\mathcal{Q}_{t}^{-1}$ como un factor de normalización para que $\|\Psi(t)\|=1$. Se tienen tambien las siguientes desigualdades,

$$
\frac{1}{\lambda_{1}+\lambda_{2}} \leq\left\|\frac{\hat{g}}{\left(\frac{p^{2}}{2}+E(t)\right)^{1 / 2}}\right\|^{2} \leq \frac{1}{\lambda_{1}}, \quad \forall t \in \mathbb{R}
$$

y

$$
\begin{aligned}
\mathcal{Q}_{t} & =\left\|\left(\frac{p^{2}}{2}+E(t)\right)^{-1}\left(c(t) \hat{g}_{\rho}+\hat{g}\right)\right\| \\
& \geq\left(1-\frac{\lambda_{2}}{\lambda_{1}}\right)\left\|\left(\frac{p^{2}}{2}+E_{0}\right)^{-1} \hat{g}\right\|, \quad \forall t \in \mathbb{R} .
\end{aligned}
$$


Demostración. Supongamos que $\Psi(t)$ es un vector propio con valor propio $-E(t), E(t)>0$, es decir,

$$
H_{\nu}(t) \Psi(t)=-E(t) \Psi(t)
$$

Para simplificar la notación, escribimos $E$ y $\Psi$ en lugar de $E(t)$ y $\Psi(t)$. La ecuación anterior es equivalente a

$$
-\frac{1}{2} \Delta \Psi-\lambda_{1}(g, \Psi) g-\lambda_{2}\left(g_{\rho}, \Psi\right) g_{\rho}=-E \Psi
$$

Aplicamos transformada de Fourier a ambos lados para obtener

$$
\frac{1}{2} p^{2} \hat{\Psi}(p)-\lambda_{1}(g, \Psi) \hat{g}(p)-\lambda_{2}\left(g_{\rho}, \Psi\right) \hat{g}_{\rho}(p)=-E \hat{\Psi}(p), \quad \forall p \in \mathbb{R}^{n}
$$

donde $p^{2}:=p_{1}^{2}+\cdots+p_{n}^{2}$ para $p=\left(p_{1}, \cdots, p_{n}\right) \in \mathbb{R}^{n}$. Entonces

$$
\hat{\Psi}=\lambda_{1}(g, \Psi) \frac{\hat{g}}{\frac{1}{2} p^{2}+E}+\lambda_{2}\left(g_{\rho}, \Psi\right) \frac{\hat{g}_{\rho}}{\frac{1}{2} p^{2}+E}
$$

Usando la expresión de $\hat{\Psi}$ dada en (2.21) y considerando que $(\hat{g}, \hat{\Psi})=(g, \Psi)$ y $\left(\hat{g}_{\rho}, \hat{\Psi}\right)=\left(g_{\rho}, \Psi\right)$, obtenemos el sistema de ecuaciones

$$
\begin{aligned}
& (\hat{g}, \hat{\Psi})=\lambda_{1}(\hat{g}, \hat{\Psi})\left(\hat{g}, \frac{\hat{g}}{\frac{1}{2} p^{2}+E}\right)+\lambda_{2}\left(\hat{g}_{\rho}, \hat{\Psi}\right)\left(\hat{g}, \frac{\hat{g}_{\rho}}{\frac{1}{2} p^{2}+E}\right) \\
& \left(\hat{g}_{\rho}, \hat{\Psi}\right)=\lambda_{1}(\hat{g}, \hat{\Psi})\left(\hat{g}_{\rho}, \frac{\hat{g}}{\frac{1}{2} p^{2}+E}\right)+\lambda_{2}\left(\hat{g}_{\rho}, \hat{\Psi}\right)\left(\hat{g}_{\rho}, \frac{\hat{g}_{\rho}}{\frac{1}{2} p^{2}+E}\right)
\end{aligned}
$$

Que en notación matricial se expresa como

$$
\left[\begin{array}{cc}
\lambda_{1}\left(\hat{g}, \hat{g}\left(\frac{1}{2} p^{2}+E\right)^{-1}\right)-1 & \lambda_{2}\left(\hat{g}, \hat{g}_{\rho}\left(\frac{1}{2} p^{2}+E\right)^{-1}\right) \\
\lambda_{1}\left(\hat{g}_{\rho}, \hat{g}\left(\frac{1}{2} p^{2}+E\right)^{-1}\right) & \lambda_{2}\left(\hat{g}_{\rho}, \hat{g}_{\rho}\left(\frac{1}{2} p^{2}+E\right)^{-1}\right)-1
\end{array}\right]\left[\begin{array}{c}
(\hat{g}, \hat{\Psi}) \\
\left(\hat{g}_{\rho}, \hat{\Psi}\right)
\end{array}\right]=0 .
$$

Notemos que

$$
\left(\hat{g}, \hat{g}\left(\frac{1}{2} p^{2}+E\right)^{-1}\right)=\left\|\hat{g}\left(\frac{1}{2} p^{2}+E\right)^{-1 / 2}\right\|^{2}
$$

y 


$$
\left(\hat{g}_{\rho}, \hat{g}_{\rho}\left(\frac{1}{2} p^{2}+E\right)^{-1}\right)=\left\|\hat{g}_{\rho}\left(\frac{1}{2} p^{2}+E\right)^{-1 / 2}\right\|^{2}
$$

Expresamos (2.23) como

$$
\left[\begin{array}{cc}
\lambda_{1}\left\|\hat{g}\left(\frac{1}{2} p^{2}+E\right)^{-1 / 2}\right\|^{2}-1 & \lambda_{2}\left(\hat{g}, \hat{g}_{\rho}\left(\frac{1}{2} p^{2}+E\right)^{-1}\right) \\
\lambda_{1}\left(\hat{g}_{\rho}, \hat{g}\left(\frac{1}{2} p^{2}+E\right)^{-1}\right) & \lambda_{2}\left\|\hat{g}_{\rho}\left(\frac{1}{2} p^{2}+E\right)^{-1 / 2}\right\|^{2}-1
\end{array}\right]\left[\begin{array}{c}
(\hat{g}, \hat{\Psi}) \\
\left(\hat{g}_{\rho}, \hat{\Psi}\right)
\end{array}\right]=0
$$

Además,

$$
\begin{aligned}
& \left(\hat{g}, \hat{g}_{\rho}\left(\frac{1}{2} p^{2}+E\right)^{-1}\right)\left(\hat{g}_{\rho}, \hat{g}\left(\frac{1}{2} p^{2}+E\right)^{-1}\right) \\
= & \left(\hat{g}\left(\frac{1}{2} p^{2}+E\right)^{-1 / 2}, \hat{g}_{\rho}\left(\frac{1}{2} p^{2}+E\right)^{-1 / 2}\right)\left(\hat{g}_{\rho}\left(\frac{1}{2} p^{2}+E\right)^{-1 / 2}, \hat{g}\left(\frac{1}{2} p^{2}+E\right)^{-1 / 2}\right) \\
\quad & \left|\left(\hat{g}\left(\frac{1}{2} p^{2}+E\right)^{-1 / 2}, \hat{g}_{\rho}\left(\frac{1}{2} p^{2}+E\right)^{-1 / 2}\right)\right|^{2}=\left|\left(\hat{g}, \hat{g}_{\rho}\left(\frac{1}{2} p^{2}+E\right)^{-1}\right)\right|^{2}
\end{aligned}
$$

En consecuencia, el sistema anterior tiene solución no trivial si, y solo si, el determinante de la matriz anterior es cero, esto es, si se cumple

$$
\begin{array}{r}
\left(1-\lambda_{2}\left\|\hat{g}_{\rho}\left(\frac{1}{2} p^{2}+E\right)^{-1 / 2}\right\|^{2}\right)\left(1-\lambda_{1}\left\|\hat{g}\left(\frac{1}{2} p^{2}+E\right)^{-1 / 2}\right\|^{2}\right) \\
=\lambda_{1} \lambda_{2}\left|\left(\hat{g}_{,} \hat{g}_{\rho}\left(\frac{1}{2} p^{2}+E\right)^{-1}\right)\right|^{2}
\end{array}
$$

Además, para $t \in \mathbb{R}$

$$
\begin{aligned}
\hat{g}_{\rho}(p) & =(2 \pi)^{-n / 2} \int_{\mathbb{R}^{n}} e^{-i<p, x>} g(x-\rho(t)) d x \\
& =(2 \pi)^{-n / 2} \int_{\mathbb{R}^{n}} e^{-i<p, y+\rho(t)>} g(y) d y \\
& =e^{-i<p, \rho(t)>} \hat{g}(p), \quad \forall p \in \mathbb{R}^{n} .
\end{aligned}
$$

De esta manera se tiene que

$$
\left\|\frac{\hat{g}_{\rho}}{\left(\frac{1}{2} p^{2}+E\right)^{1 / 2}}\right\|=\left\|\frac{\hat{g}}{\left(\frac{1}{2} p^{2}+E\right)^{1 / 2}}\right\|
$$


Así, la ecuación (2.25) es equivalente a

$$
\begin{array}{r}
\left(1-\lambda_{2}\left\|\hat{g}\left(\frac{1}{2} p^{2}+E\right)^{-1 / 2}\right\|^{2}\right)\left(1-\lambda_{1}\left\|\hat{g}\left(\frac{1}{2} p^{2}+E\right)^{-1 / 2}\right\|^{2}\right) \\
=\lambda_{1} \lambda_{2}\left|\left(\hat{g}, \hat{g}_{\rho}\left(\frac{1}{2} p^{2}+E\right)^{-1}\right)\right|^{2}
\end{array}
$$

Mostraremos que $-E(t)$ satisface $-E(t) \leq-Q_{1}<-Q_{2}$. Del corolario 3.5, sabemos que $-Q_{1}$ es negativo. Tomemos $E>0$ independiente de $t$.

Definamos

$$
D_{E}(t)=\left(\lambda_{1}+\lambda_{2}\right) a+\lambda_{1} \lambda_{2}\left(f(t)-a^{2}\right)
$$

donde

$$
a=\left\|\frac{\hat{g}}{\left(\frac{1}{2} p^{2}+E\right)^{1 / 2}}\right\|^{2}
$$

y

$$
f(t)=\left|\left(\hat{g}, \hat{g}_{\rho}\left(\frac{1}{2} p^{2}+E\right)^{-1}\right)\right|^{2} .
$$

Observemos que en la última ecuación, aunque $E$ es independiente de $t$, la dependencia en $\hat{g}_{\rho}$ de $t$, implica la de $f$. La función $D_{E}(t)$ viene de desarrollar (2.28) de tal manera que la ecuación (2.28) se cumple si $D_{E}(t)=1$.

Nótese que

$$
f(t)-a^{2} \leq 0, \quad \forall t \in \mathbb{R}
$$

pues, por la desigualdad de Cauchy-Schwarz y por (2.27),

$$
\begin{aligned}
\left|\left(\hat{g}, \frac{\hat{g}_{\rho}}{\frac{1}{2} p^{2}+E}\right)\right| & \leq\left\|\frac{\hat{g}}{\left(\frac{1}{2} p^{2}+E\right)^{1 / 2}}\right\|\left\|\frac{\hat{g}_{\rho}}{\left(\frac{1}{2} p^{2}+E\right)^{1 / 2}}\right\| \\
& =\left\|\frac{\hat{g}}{\left(\frac{1}{2} p^{2}+E\right)^{1 / 2}}\right\|^{2}
\end{aligned}
$$

Por tanto,

$$
\sup _{t \in \mathbb{R}} D_{E}(t) \leq\left(\lambda_{1}+\lambda_{2}\right) a
$$

Además, desarrollando $f(t)$ se obtiene,

$$
f(t)=\left|\int_{\mathbf{R}^{n}} e^{-i<p, \rho(t)>} \frac{|\hat{g}(p)|^{2}}{\frac{1}{2} p^{2}+E} d p\right|^{2}=|\hat{s}(\rho(t))|^{2}
$$


con $s$ en $L^{1}\left(\mathbb{R}^{n}\right)$ y definida como

$$
s(p)=\frac{|\hat{g}(p)|^{2}}{\frac{1}{2} p^{2}+E}, \forall p \in \mathbb{R}^{n}
$$

Según la definición de $\rho, \lim _{t \rightarrow \pm \infty}|\rho(t)|=\infty$, y del lema de RiemannLesbesgue se obtiene $\lim _{t \rightarrow \pm \infty}|h(\rho(t))|=0$. Se sigue que,

$$
\lim _{t \rightarrow \pm \infty} f(t)=0
$$

Entonces se cumple

$$
\inf _{t \in \mathbb{R}} D_{E}(t)=D_{E}( \pm \infty)=\left(\lambda_{1}+\lambda_{2}\right) a-\lambda_{1} \lambda_{2} a^{2}
$$

Por tanto, $-E$ es valor propio de $H_{\nu}(t)$, si y sólo si,

$$
\left(\lambda_{1}+\lambda_{2}\right) a-\lambda_{1} \lambda_{2} a^{2} \leq 1 \leq \sup _{t \in \mathbb{R}} D_{E}(t) \leq\left(\lambda_{1}+\lambda_{2}\right) a .
$$

También se tiene

$$
\begin{aligned}
\sup _{a}\left(\lambda_{1}+\lambda_{2}\right) a-\lambda_{1} \lambda_{2} a^{2} & =\left(\left(\lambda_{1}+\lambda_{2}\right) a-\lambda_{1} \lambda_{2} a^{2}\right) l_{a=\left(\lambda_{1}+\lambda_{2}\right) / 2 \lambda_{1} \lambda_{2}} \\
& =\frac{\left(\lambda_{1}+\lambda_{2}\right)^{2}}{4 \lambda_{1} \lambda_{2}}>1 .
\end{aligned}
$$

La primera desigualdad en (2.36) es equivalente a la desigualdad

$$
0 \leq\left(a-\frac{1}{\lambda_{1}}\right)\left(a-\frac{1}{\lambda_{2}}\right)
$$

misma que tiene solución

$$
a \leq \frac{1}{\lambda_{1}} \quad \text { ó } \quad a \geq \frac{1}{\lambda_{2}} \text {. }
$$

Observemos que $\left(\lambda_{1}+\lambda_{2}\right) a-\lambda_{1} \lambda_{2} a^{2}=1$ si y sólo si $a=1 / \lambda_{1}$ ó $a=1 / \lambda_{2}$. Ahora considerando la desigualdad $1 \leq\left(\lambda_{1}+\lambda_{2}\right) a$ en (2.36), junto con (2.38), se obtiene que los valores de $a$ en los que (2.36) se cumplen, son:

$$
\frac{1}{\lambda_{1}+\lambda_{2}} \leq a \leq \frac{1}{\lambda_{1}} \quad \text { ó } a \geq \frac{1}{\lambda_{2}}
$$


Sabemos de (2.13) que a es monótona decreciente como función de $E$ entonces, se cumple

$$
-E_{0} \leq-E(t) \leq-Q_{1}
$$

ó

$$
-Q_{2} \leq-E(t)<0 .
$$

De la demostración de la proposición 2.1 y el corolario 2.1 se sigue que

$$
\begin{aligned}
& \left\|\frac{\hat{g}}{\left(\frac{1}{2} p^{2}+Q_{1}\right)^{1 / 2}}\right\|^{2}=\frac{1}{\lambda_{1}} \\
& \left\|\frac{\hat{g}}{\left(\frac{1}{2} p^{2}+E_{0}\right)^{1 / 2}}\right\|^{2}=\frac{1}{\lambda_{1}+\lambda_{2}}
\end{aligned}
$$

De (2.30), (2.39), (2.40) y (2.41), se obtiene (2.17).

Ahora probamos que hay solamente un valor propio en el intervalo $\left[-E_{0},-Q_{1}\right]$ y es de multiplicidad 1. Sea $h(t)$ una función continua de $\mathbb{R}$ a $\mathbb{R}^{n}$ tal que $h(t)=\rho(t)$ si $|t|>t_{0}$ para algún $t_{0}>0$ y $h(0)=\overrightarrow{0} \in \mathbb{R}^{n}$. Ahora consideremos el operador $\bar{H}_{\nu}(t)$ definido como $H_{\nu}(t)$ con $h$ en lugar de $\rho$. Aplicando el corolario (2.1) obtenemos que

$$
\operatorname{dim} \bar{P}_{\left[-E_{0},-Q_{1}\right]}(0)=1
$$

donde $\bar{P}_{A}(t)$ es el proyector espectral de $\bar{H}_{\nu}(t)$ asociado al conjunto de Borel $A$. Por el teorema (1.9) se obtiene

$$
\operatorname{dim} \bar{P}_{\left[-E_{0},-Q_{1}\right]}(t)=1, \forall t \in \mathbb{R} .
$$

Por (2.43) se tiene que si $|t|>t_{0}$,

$$
\operatorname{dim} P_{\left[-E_{0},-Q_{1}\right]}(t)=\operatorname{dim} \bar{P}_{\left[-E_{0,}-Q_{1}\right]}(t)=1,
$$

donde $P_{A}$ es el proyector espectral de $H_{\nu}(t)$ asociado con el conjunto de Borel $A$. Aplicando el teorema (1.9) otra vez, se concluye que

$$
\operatorname{dim} P_{\left[-E_{0},-Q_{1}\right]}(t)=1, \forall t \in \mathbb{R} .
$$

Dado que (2.24) tiene solución no trivial, se deduce que es equivalente a

$$
\lambda_{1}\left(\hat{g}_{\rho}, \hat{g}\left(p^{2} / 2+E\right)^{-1}\right)(\hat{g}, \hat{\Psi})+\left(\lambda_{2}\left\|\hat{g}_{\rho}\left(p^{2} / 2+E\right)^{-1 / 2}\right\|^{2}-1\right)\left(\hat{g}_{\rho}, \hat{\Psi}\right)=0 .
$$


De donde se obtiene

$$
\left(\hat{g}_{\rho}, \hat{\Psi}\right)=\left(g_{\rho}, \Psi\right)=\frac{\lambda_{1}(g, \Psi)\left(\hat{g}_{\rho}, \hat{g}\left(p^{2} / 2+E(t)\right)^{-1}\right)}{1-\lambda_{2}\left\|\hat{g}\left(p^{2} / 2+E(t)\right)^{-1 / 2}\right\|^{2}} .
$$

Sustituyendo la igualdad (2.47) en (2.21), se obtiene

$$
\hat{\Psi}=\lambda_{1}(g, \Psi)\left(p^{2} / 2+E(t)\right)^{-1}\left(c(t) \hat{g}_{\rho}+\hat{g}\right) .
$$

donde $c(t)$ esta definido como en (2.16). Aplicando transformada inversa de Fourier, $\mathcal{F}^{-1}$, en (2.48), obtenemos (2.15) con $\mathcal{Q}_{t}$ definido como en 2.18 .

\subsection{Caracterización del estado fundamental}

Probamos algunas estimaciones respecto a la variable temporal del estado fundamental y su valor propio correspondiente del operador $H_{v}(t)$. Esto nos permite estudiar la probabilidad de transición del estado inicial.

Teorema 2.2. Sean $g$ y $\rho$ definidos en (2.2)-(2.4). Consideremos el operador definido en (2.1). Las siguientes relaciones son válidas para su estado fundamental $\psi(t)$ y el correspondiente valor propio $-E(t)$ : para $k=1,2$,

$$
\begin{gathered}
\left|\frac{d^{k}}{d t^{k}} E\left(\frac{t}{v}\right)\right| \leq \frac{C_{M}}{t^{2}} \quad \text { y } \quad\left\|\frac{d^{k}}{d t^{k}} \Psi\left(\frac{t}{v}\right)\right\| \leq \frac{C_{M}}{t^{2}} \quad \text { si }|t|>T_{M} \\
\sup _{t \in \mathbb{R}}\left|\frac{d^{k}}{d t^{k}} E\left(\frac{t}{v}\right)\right| \leq C_{M} \text { y } \quad \sup _{t \in \mathbb{R}}\left\|\frac{d^{k}}{d t^{k}} \Psi\left(\frac{t}{v}\right)\right\| \leq C_{M}
\end{gathered}
$$

donde las constantes $C_{M}$ y $T_{M}$ solo dependen de la $M$ tomada como en (2.8).

Demostración. Las desigualdades (2.50) se siguen directamente de (2.5)(2.7) y de las ecuaciones de abajo.

Nos concentramos ahora en probar (2.49). Primero para

$$
\left|\frac{d^{k}}{d t^{k}} E\left(\frac{t}{v}\right)\right| \leq \frac{C_{M}}{t^{2}} \quad \text { si }|t|>T_{M} .
$$

No hay pérdida de generalidad si tomamos $\rho(t)$ de la forma

$$
\rho(t):=\left(\rho_{1}(t), 0,0, \cdots, 0\right) .
$$


Notemos que

$\frac{1}{2} \cdot|t| \leq\left|\rho_{1}\left(\frac{t}{v}\right)\right| \quad$ si $\quad T_{M}<|t| ; \quad \mathrm{y}, \quad\left|\frac{d^{k}}{d t^{k}} \rho_{1}\left(\frac{t}{v}\right)\right| \leq C_{1, M} \quad \forall t \in \mathbb{R}, k=1,2$.

Considerando que $H_{v}(t / v) \Psi(t / v)=-E(t / v) \Psi(t / v)$ con $\|\Psi\|=1$, se tiene que

$$
\left(\Psi(t / v), H_{v}(t / v) \Psi(t / v)\right)=-E(t / v), \quad \forall t \in \mathbb{R}
$$

y luego,

$$
\begin{aligned}
v^{-1} E^{\prime}(t / v) & :=-\frac{d}{d t} E\left(\frac{t}{v}\right)=\frac{d}{d t}\left(\Psi(t / v), H_{v}(t / v) \Psi(t / v)\right) \\
& =\left(\Psi^{\prime}(t / v), H_{v}(t / v) \Psi(t / v)\right)+\left(\Psi(t / v), H_{v}^{\prime}(t / v) \Psi(t / v)\right) \\
& =\left(\Psi^{\prime}(t / v), E(t / v) \Psi(t / v)\right)+\left(\Psi(t / v), H_{v}^{\prime}(t / v) \Psi(t / v)+H_{v}(t / v) \Psi^{\prime}(t / v)\right) \\
& =0+\left(\Psi(t / v), H_{v}^{\prime}(t / v) \Psi(t / v)\right)+\left(H_{v}(t / v) \Psi(t / v), \Psi^{\prime}(t / v)\right) \\
& =v^{-1}\left(\Psi\left(\frac{t}{v}\right), H_{v}^{\prime}\left(\frac{t}{v}\right) \Psi\left(\frac{t}{v}\right)\right)
\end{aligned}
$$

donde hemos usado el hecho de que $H_{v}$ es autoadjunto y que $\left(\Psi(t), \Psi^{\prime}(t)\right)=0$. De la ecuación (2.55) se obtiene una expresión más explícita para $\frac{d}{d t} E\left(\frac{t}{v}\right)$ $\frac{i \lambda_{2}}{v}\left[\left(\vec{p} \cdot \rho^{\prime}(t / v) \hat{g}_{\rho}, \hat{\Psi}(t / v)\right)\left(\hat{\Psi}(t / v), \hat{g}_{\rho}\right)-\left(\hat{\Psi}(t / v), \vec{p} \cdot \rho^{\prime}(t / v) \hat{g}_{\rho}\right)\left(\hat{g}_{\rho}, \hat{\Psi}(t / v)\right)\right]$

donde $\cdot$ denota el producto escalar usual en $\mathbb{R}^{n}$. De (2.53) y (2.15)-(2.18) se tiene

$$
\begin{aligned}
& v^{-1}\left|\left(\vec{p} \cdot \rho^{\prime}(t / v) \hat{g}_{\rho}, \hat{\Psi}(t / v)\right)\right| \leq d_{1}|c(t / v)| \\
& \quad+\left.d_{2}\left|v^{-1} \int e^{i \vec{p} \cdot \rho(t / v)} \vec{p} \cdot \rho(t / v)\right| \hat{g}(\vec{p})\right|^{2}\left(p^{2} / 2+E(t / v)\right)^{-1} d^{n} p \mid
\end{aligned}
$$

El segundo término de la desigualdad previa es igual a

$$
\begin{aligned}
& \left.\frac{d_{2}}{\rho_{1}^{2}(t / v)}\left|v^{-1} \rho_{1}^{\prime}(t / v) \int \frac{\partial^{2}}{\partial p_{1}^{2}} e^{i \vec{p} \cdot \rho(t / v)} p_{1}\right| \hat{g}(\vec{p})\right|^{2}\left(p^{2} / 2+E(t / v)\right)^{-1} d^{n} p \mid= \\
& \left.\frac{1}{\rho_{1}^{2}(t / v)}\left|v^{-1} \rho_{1}^{\prime}(t / v) \int e^{i \vec{p} \cdot \rho(t / v)} \frac{\partial^{2}}{\partial p_{1}^{2}} p_{1}\right| \hat{g}(\vec{p})\right|^{2}\left(p^{2} / 2+E(t / v)\right)^{-1} d^{n} p\left|\leq \frac{d_{3}}{t^{2}},\right| t \mid>T_{M} .
\end{aligned}
$$


donde hemos usado (2.53) y la hipótesis sobre $g$. De manera similar, podemos probar usando (2.16) y (2.17) que

$$
\left|c\left(\frac{t}{v}\right)\right| \leq \frac{C_{M}}{t^{2}}, \quad|t|>T_{M}
$$

Obtenemos (2.51) de (2.55)-(2.59). Ahora probemos (2.49) para $\frac{d}{d t} \Psi(t / v)$. De (2.15)-(2.18) podemos observar que

$$
\frac{d}{d t} \Psi(t / v)=\mathcal{F}^{-1}\left[\frac{d}{d t} \mathcal{Q}_{t / v}^{-1} \cdot G\left(\frac{t}{v}\right)+\mathcal{Q}_{t / v}^{-1} \frac{d}{d t} G\left(\frac{t}{v}\right)\right]
$$

donde $\mathcal{F}^{-1}$ denota la transformada inversa de Fourier y

$$
G(t):=\left(p^{2} / 2-E(t)\right)^{-1}\left(c(t) \hat{g}_{\rho}+\hat{g}\right) .
$$

La derivada de $\mathcal{Q}_{t / v}^{-1}$ se comporta para $|t|>T_{M}$ como la derivada de $E(t / v)$ y de $c(t / v)$. Aqui usamos (2.18). La norma $L^{2}$ de la derivada de $G(t / v)$ también se comporta de esta manera. Usando (2.17) y una estimación como en (2.58) podemos ver que la primera derivada $c(t / v)$ se comporta como $O\left(1 / t^{2}\right)$. Dado que hemos visto ya que que $\frac{d}{d t} E(t / v)$ es de éste orden, obtenemos (2.49) para $k=1$. Notemos ahora que

$$
\begin{aligned}
-\frac{d^{2}}{d t^{2}} E\left(\frac{t}{v}\right) & =\left(\Psi\left(\frac{t}{v}\right),\left[\frac{d^{2}}{d t^{2}} H_{v}\left(\frac{t}{v}\right)\right] \Psi\left(\frac{t}{v}\right)\right) \\
& +\left(\frac{d}{d t} \Psi\left(\frac{t}{v}\right),\left[\frac{d}{d t} H_{v}\left(\frac{t}{v}\right)\right] \Psi\left(\frac{t}{v}\right)\right) \\
& +\left(\Psi\left(\frac{t}{v}\right),\left[\frac{d}{d t} H_{v}\left(\frac{t}{v}\right)\right] \frac{d}{d t} \Psi\left(\frac{t}{v}\right)\right)
\end{aligned}
$$

De (2.30) y la hipótesis sobre la función $g$ se obtiene

$$
\left\|\frac{d^{k}}{d t^{k}} H_{v}\left(\frac{t}{v}\right)\right\| \leq C, k=1,2 .
$$

De la desigualdad previa (2.62) y la estimación obtenida para $\frac{d}{d t} \Psi(t / v)$ se deduce que los últimos términos del lado derecho de la ecuación (2.61) son 
de orden 1/ $t^{2}$. Para estimar el primer término de (2.61) se puede proceder como arriba: notemos que

$$
\frac{d^{2}}{d t^{2}} H_{v}(t)=\mathcal{F}^{-1} \sum_{r+s=2}\left(\frac{d^{r}}{d t^{r}} e^{-i \rho(t) \cdot \vec{p}} \hat{g}, \cdot\right) \frac{d^{s}}{d t^{s}} e^{-i \rho(t) \cdot \vec{p}} \hat{g} \mathcal{F}
$$

Además, si $r=1,2$,

$$
\frac{d^{r}}{d t^{r}} e^{-i \rho(t) \cdot \vec{p}}=\frac{d^{r}}{d t^{r}} e^{-i \rho(t) \cdot \vec{p}} \sum_{\beta_{1}+2 \beta_{2}=r}(i)^{\alpha_{\beta_{1} \beta_{2}}} d_{\beta_{1} \beta_{2}}\left(\rho^{\prime} \cdot \vec{p}\right)^{\beta_{1}}\left(\rho^{\prime \prime} \cdot \vec{p}\right)^{\beta_{2}}
$$

Aqui $\beta_{i}, i=1,2$, son enteros no negativos. Por tanto, como en (2.57) $-(2.59)$ con (2.15)-(2.18) y (2.53) se obtiene

$$
\begin{aligned}
&\left|\left(\hat{g}\left(\frac{d^{r}}{d t^{r}} e^{-i \rho(t / v) \cdot \vec{p}}\right), \hat{\Psi}\left(\frac{t}{v}\right)\right)\right| \leq d_{1}\left|c\left(\frac{t}{v}\right)\right| \\
&+\left.\frac{d_{2}}{t^{2}}\left|\int e^{i \rho(t / v) \cdot \vec{p}} \frac{\partial^{2}}{\partial p_{1}^{2}} e^{-i \rho(t / v) \cdot \vec{p}} \frac{d^{r}}{d t^{r}} e^{-i \rho(t / v) \cdot \vec{p}}\right| \hat{g}(\vec{p})\right|^{2}\left(\frac{p^{2}}{2}+E\left(\frac{t}{v}\right)\right)^{-1} d^{n} p\left|\leq \frac{d}{t^{2}}, \quad\right| t \mid>T_{M},
\end{aligned}
$$

donde hemos usado (2.6)-(2.7), (2.64), y la hipótesis sobre la función $g$. Usando (2.61)-(2.65) probamos que $\frac{d^{2}}{d t^{2}} E\left(\frac{t}{v}\right)$ se comporta como $O\left(1 / t^{2}\right)$, para $|t|>T_{M}$. Un argumento muy similar como la dada para la primera derivada de $\Psi(t / v)$ puede ser usado para probar que $\frac{d^{2}}{d t^{2}} \Psi\left(\frac{t}{v}\right)$ es de orden $1 / t^{2}$. Con ésto se prueba el teorema. 


\section{Capítulo 3}

\section{Sobre los valores propios de un operador dependiente del tiempo}

En este capítulo consideramos un hamiltoniano más complejo respecto al estudiado en capítulos anteriores, generalizando además algunos resultados obtenidos previamente por [Arr1].

\subsection{La definición del operador}

En esta sección definimos el hamiltoniano con el cual trabajaremos a lo largo de este capítulo.

Para cada $t \in \mathbb{R}$, sea el operador

$$
H(t)=-\frac{1}{2} \Delta-\lambda_{1} V_{1}-\lambda_{2} V_{1, \rho}-\mu_{1} V_{2}-\mu_{2} V_{2, \rho}
$$

donde, como en el capítulo anterior, $\Delta$ es el operador de Laplace en $\mathbb{R}^{n}$ y, $\lambda_{i}$ y $\mu_{i}$, con $i=1,2$, son constantes reales positivas. Consideramos el operador $H(t)$ definido sobre $L^{2}\left(\mathbb{R}^{n}, d x\right)$, con dominio $H^{2}\left(\mathbb{R}^{n}\right)$. Los operadores $V_{i}$ estan definidos por

$$
V_{i} \varphi=\left(g_{i}, \varphi\right) g_{i}, \quad \forall \varphi \in L^{2}\left(\mathbb{R}^{n}, d x\right), \quad i=1,2,
$$

donde las funciones $g_{i},(i=1,2)$, son funciones no nulas de $L^{2}\left(\mathbb{R}^{n}, d x\right)$, y son tales que

$$
|p| \hat{g}_{i}(p) \in L^{2}\left(\mathbb{R}^{n}, d x\right) .
$$


Además,

$$
V_{i, \rho} \varphi=\left(g_{i, \rho}, \varphi\right) g_{i, \rho}, \quad \text { con } \quad g_{i, \rho}(x)=g_{i}(x-\rho(t)) .
$$

En la definición de $g_{i, \rho}, \rho(t)$ es una función continua de $\mathbb{R}$ a $\mathbb{R}^{n}$, que para simplificar la notación suponemos que $\rho(0)=\overrightarrow{0} \in \mathbb{R}^{n}$ y

$$
\lim _{|t| \rightarrow \infty}|\rho(t)|=\infty
$$

\subsection{El teorema}

Por el teorema de Weyl se tiene que el espectro esencial de $H(t)$, para cada $t \in \mathbb{R}$, coincide con el espectro esencial de $\Delta,[0, \infty)$. Así, el espectro discreto de $H(t)$ es subconjunto de $(-\infty, 0)$.

En el capítulo anterior vimos que para una función $g \in L^{2}\left(\mathbb{R}^{n}, d x\right)$ no nula, existe una constante positiva suficientemente grande $\alpha_{0}$ tal que el operador

$$
-\frac{1}{2} \Delta-\alpha(g, \cdot) g
$$

tiene un único valor propio negativo $-E_{\alpha}:=E$ para $\alpha \geq \alpha_{0}$. De hecho, probamos que $-E$ es un valor propio negativo si y sólo si

$$
\frac{1}{\alpha}=\left\|\left(-\frac{1}{2} \Delta+E\right)^{-\frac{1}{2}} g\right\|^{2} \text {. }
$$

También vimos que para $g$ fijo, la función

$$
h(E)=\left\|\left(-\frac{1}{2} \Delta+E\right)^{-\frac{1}{2}} g\right\|^{2}
$$

es estrictamente decreciente. Además, la dimensión del espacio correspondiente a $E$ es 1 .

Teorema 3.1. Sean $g_{i} \in L^{2}\left(\mathbb{R}^{n}, d x\right)$ y $\hat{g}_{i}$ funciones no negativas, tales que $|p| \hat{g}_{i} \in L^{2}\left(\mathbb{R}^{n}, d x\right)$ para $i=1,2$. Supongamos que las constantes $\lambda_{i}, \mu_{i}$ cumplen $\lambda_{1}>\mu_{1}+\mu_{2}>\mu_{1}>\lambda_{2}>\mu_{2}>0$. Además, asumimos que $0<E_{0}(2)<E_{1}$ y $0<E_{\lambda_{2}}<E_{\mu_{1}}$, donde $-E_{1},-E_{0}(2),-E_{\lambda_{2}}$ y $-E_{\mu_{1}}$ son los valores propios de los estados fundamentales de los operadores

$$
-\frac{1}{2} \Delta-\lambda_{1} V_{1}, \quad-\frac{1}{2} \Delta-\left(\mu_{1}+\mu_{2}\right) V_{2}, \quad-\frac{1}{2} \Delta-\lambda_{2} V_{1}, \quad-\frac{1}{2} \Delta-\mu_{1} V_{2},
$$

respectivamente. Entonces, los siguientes enunciados se cumplen 
1. $\mathrm{Si}-E(t)$ es valor propio correspondiente al estado fundamental del operador $H(t)$, con $t \in \mathbb{R}$, entonces

$$
-E(t) \in\left[-E_{0},-E_{\infty}\right),
$$

donde $-E(0)$ y $-E_{\infty}$ son estados fundamentales de $H(0)$ y $-\frac{1}{2} \Delta-$ $\lambda_{1} V_{1}-\mu_{1} V_{2}$, respectivamente.

2. En el intervalo $\left(-E_{\infty},-E_{1}\right]$ no hay valores propios de $H(t)$, para cualquier $t \in \mathbb{R}$.

\subsection{La demostración}

En esta sección desarrollamos la demostración del teorema 3.1, siguiendo la técnica usada en el teorema 2.1. Para ello, probamos previamente los siguientes lemas.

Lema 3.1. El espectro discreto del operador autoadjunto $H(t)$, es un subconjunto del intervalo $(-\infty, 0)$.

Demostración. Dado que $-\Delta$ es un operador autoadjunto en $H^{2}\left(\mathbb{R}^{n}\right)$ y $V_{i}, V_{i, \rho}$ con $i=1,2$, son operadores autodajuntos compactos, el teorema de Katto-Rellich asegura que $H(t), t \in \mathbb{R}$, es autoadjunto. Puesto que $-\Delta$ tiene espectro esencial $[0, \infty)$, al aplicar el teorema de Weyl se deduce que el espectro discreto de $H(t)$ es subconjunto de $(-\infty, 0)$.

Cuando $t \rightarrow \pm \infty, g_{i, \rho}$ se anula (por el lema de Riemann-Lebesgue), y por tanto $V_{i, \rho}=0$ en este caso. Ésto justifica la definición de $H( \pm \infty)$ que consideramos en el siguiente teorema.

Lema 3.2. El valor propio $-E_{0}$, correspondiente al estado fundamental del operador

$$
H(0)=-\frac{1}{2} \Delta-\left(\lambda_{1}+\lambda_{2}\right) V_{1}-\left(\mu_{1}+\mu_{2}\right) V_{2}
$$

y el valor propio $-E_{\infty}$, correspondiente al estado fundamental del operador

$$
H( \pm \infty):=-\frac{1}{2} \Delta-\lambda_{1} V_{1}-\mu_{1} V_{2}
$$

son negativos y, se cumple la desigualdad $-E_{0}<-E_{\infty}$. 
Demostración. Supongamos que $-E(t):=-E$ es un valor propio de $H(t)$ para algún $t>0$. Entonces existe algún vector propio no nulo $\psi(t)=$ $\psi \in L^{2}\left(\mathbb{R}^{n}\right)$ tal que

$$
H(t) \psi=-E \psi
$$

No escribimos la dependencia de $E$ y de $\psi$ en $t$ para simplificar la notación. Ahora, como en el capítulo anterior, aplicamos la transformada de Fourier en ambos lados de la ecuación (3.5), para obtener

$$
\frac{p^{2}}{2} \hat{\psi}-\lambda_{1}\left(g_{1}, \psi\right) \hat{g}_{1}-\lambda_{2}\left(g_{1, \rho}, \psi\right) \hat{g}_{1, \rho}-\mu_{1}\left(g_{2}, \psi\right) \hat{g}_{2}-\mu_{2}\left(g_{2, \rho}, \psi\right) \hat{g}_{2, \rho}=-E \hat{\psi}
$$

Así, una expresión para la transformada de Fourier del vector propio $\psi$, es

$$
\hat{\psi}=\lambda_{1}\left(g_{1}, \psi\right) \frac{\hat{g}_{1}}{\frac{p^{2}}{2}+E}+\lambda_{2}\left(g_{1, \rho}, \psi\right) \frac{\hat{g}_{1, \rho}}{\frac{p^{2}}{2}+E}+\mu_{1}\left(g_{2}, \psi\right) \frac{\hat{g}_{2}}{\frac{p^{2}}{2}+E}+\mu_{2}\left(g_{2, \rho}, \psi\right) \frac{\hat{g}_{2, \rho}}{\frac{p^{2}}{2}+E}
$$

Con la expresión anterior y considerando que la transformada de Fourier es un operador unitario, se obtienen las siguientes relaciones

$$
\begin{aligned}
\left(\hat{g}_{1}, \hat{\psi}\right)= & \lambda_{1}\left(\hat{g}_{1}, \hat{\psi}\right)\left(\hat{g}_{1},\left(p^{2} / 2+E\right)^{-1} \hat{g}_{1}\right)+\lambda_{2}\left(\hat{g}_{1, \rho}, \hat{\psi}\right)\left(\hat{g}_{1},\left(p^{2} / 2+E\right)^{-1} \hat{g}_{1, \rho}\right) \\
& +\mu_{1}\left(\hat{g}_{2}, \hat{\psi}\right)\left(\hat{g}_{1},\left(p^{2} / 2+E\right)^{-1} \hat{g}_{2}\right)+\mu_{2}\left(\hat{g}_{2, \rho}, \hat{\psi}\right)\left(\hat{g}_{1},\left(p^{2} / 2+E\right)^{-1} \hat{g}_{2, \rho}\right) \\
= & \lambda_{1}\left(\hat{g}_{1}, \hat{\psi}\right)\left\|\left(p^{2} / 2+E\right)^{-1 / 2} \hat{g}_{1}\right\|^{2}+\lambda_{2}\left(\hat{g}_{1, \rho}, \hat{\psi}\right)\left(\hat{g}_{1},\left(p^{2} / 2+E\right)^{-1} \hat{g}_{1, \rho}\right) \\
& +\mu_{1}\left(\hat{g}_{2}, \hat{\psi}\right)\left(\hat{g}_{1},\left(p^{2} / 2+E\right)^{-1} \hat{g}_{2}\right)+\mu_{2}\left(\hat{g}_{2, \rho}, \hat{\psi}\right)\left(\hat{g}_{1},\left(p^{2} / 2+E\right)^{-1} \hat{g}_{2, \rho}\right)
\end{aligned}
$$

$$
\begin{aligned}
\left(\hat{g}_{1, \rho}, \hat{\psi}\right)= & \lambda_{1}\left(\hat{g}_{1}, \hat{\psi}\right)\left(\hat{g}_{1, \rho},\left(p^{2} / 2+E\right)^{-1} \hat{g}_{1}\right)+\lambda_{2}\left(\hat{g}_{1, \rho}, \hat{\psi}\right)\left(\hat{g}_{1, \rho},\left(p^{2} / 2+E\right)^{-1} \hat{g}_{1, \rho}\right) \\
& +\mu_{1}\left(\hat{g}_{2}, \hat{\psi}\right)\left(\hat{g}_{1, \rho},\left(p^{2} / 2+E\right)^{-1} \hat{g}_{2}\right)+\mu_{2}\left(\hat{g}_{2, \rho}, \hat{\psi}\right)\left(\hat{g}_{1, \rho},\left(p^{2} / 2+E\right)^{-1} \hat{g}_{2, \rho}\right) \\
= & \lambda_{1}\left(\hat{g}_{1}, \hat{\psi}\right)\left(\hat{g}_{1, \rho}\left(p^{2} / 2+E\right)^{-1} \hat{g}_{1}\right)+\lambda_{2}\left(\hat{g}_{1, \rho}, \hat{\psi}\right)\left\|\left(p^{2} / 2+E\right)^{-1 / 2} \hat{g}_{1, \rho}\right\|^{2} \\
& +\mu_{1}\left(\hat{g}_{2}, \hat{\psi}\right)\left(\hat{g}_{1, \rho},\left(p^{2} / 2+E\right)^{-1} \hat{g}_{2}\right)+\mu_{2}\left(\hat{g}_{2, \rho}, \hat{\psi}\right)\left(\hat{g}_{1, \rho},\left(p^{2} / 2+E\right)^{-1} \hat{g}_{2, \rho}\right)
\end{aligned}
$$

$$
\begin{aligned}
\left(\hat{g}_{2}, \hat{\psi}\right)= & \lambda_{1}\left(\hat{g}_{1}, \hat{\psi}\right)\left(\hat{g}_{2},\left(p^{2} / 2+E\right)^{-1} \hat{g}_{1}\right)+\lambda_{2}\left(\hat{g}_{1, \rho}, \hat{\psi}\right)\left(\hat{g}_{2},\left(p^{2} / 2+E\right)^{-1} \hat{g}_{1, \rho}\right) \\
& +\mu_{1}\left(\hat{g}_{2}, \hat{\psi}\right)\left(\hat{g}_{2},\left(p^{2} / 2+E\right)^{-1} \hat{g}_{2}\right)+\mu_{2}\left(\hat{g}_{2, \rho}, \hat{\psi}\right)\left(\hat{g}_{2},\left(p^{2} / 2+E\right)^{-1} \hat{g}_{2, \rho}\right) \\
= & \lambda_{1}\left(\hat{g}_{1}, \hat{\psi}\right)\left(\hat{g}_{2},\left(p^{2} / 2+E\right)^{-1} \hat{g}_{1}\right)+\lambda_{2}\left(\hat{g}_{1, \rho}, \hat{\psi}\right)\left(\hat{g}_{2},\left(\hat{p}^{2} / 2+E\right)^{-1} \hat{g}_{1, \rho}\right) \\
& +\mu_{1}\left(\hat{g}_{2}, \hat{\psi}\right)\left\|\left(p^{2} / 2+E\right)^{-1 / 2} \hat{g}_{2}\right\|^{2}+\mu_{2}\left(\hat{g}_{2, \rho}, \hat{\psi}\right)\left(\hat{g}_{2},\left(p^{2} / 2+E\right)^{-1} \hat{g}_{2, \rho}\right)
\end{aligned}
$$




$$
\begin{aligned}
\left(\hat{g}_{2, \rho}, \hat{\psi}\right)= & \lambda_{1}\left(\hat{g}_{1}, \hat{\psi}\right)\left(\hat{g}_{2, \rho},\left(p^{2} / 2+E\right)^{-1} \hat{g}_{1}\right)+\lambda_{2}\left(\hat{g}_{1, \rho}, \hat{\psi}\right)\left(\hat{g}_{2, \rho},\left(p^{2} / 2+E\right)^{-1} \hat{g}_{1, \rho}\right) \\
& +\mu_{1}\left(\hat{g}_{2}, \hat{\psi}\right)\left(\hat{g}_{2, \rho},\left(p^{2} / 2+E\right)^{-1} \hat{g}_{2}\right)+\mu_{2}\left(\hat{g}_{2, \rho}, \hat{\psi}\right)\left(\hat{g}_{2, \rho},\left(p^{2} / 2+E\right)^{-1} \hat{g}_{2, \rho}\right) \\
= & \lambda_{1}\left(\hat{g}_{1}, \hat{\psi}\right)\left(\hat{g}_{2, \rho},\left(p^{2} / 2+E\right)^{-1} \hat{g}_{1}\right)+\lambda_{2}\left(\hat{g}_{1, \rho}, \hat{\psi}\right)\left(\hat{g}_{2, \rho},\left(p^{2} / 2+E\right)^{-1} \hat{g}_{1, \rho}\right) \\
& +\mu_{1}\left(\hat{g}_{2}, \hat{\psi}\right)\left(\hat{g}_{2, \rho},\left(p^{2} / 2+E\right)^{-1} \hat{g}_{2}\right)+\mu_{2}\left(\hat{g}_{2, \rho}, \hat{\psi}\right)\left\|\left(p^{2} / 2+E\right)^{-1 / 2} \hat{g}_{2, \rho}\right\|^{2}
\end{aligned}
$$

Las ecuaciones (3.7)-(3.10) deben cumplirse si $\psi$ es vector propio correspondiente al valor propio $-E$. Para simplificar notación hacemos

$$
\begin{aligned}
& a_{11}=\frac{1}{\lambda_{1}}-\left\|\left(p^{2} / 2+E\right)^{-1 / 2} \hat{g}_{1}\right\|^{2}, \\
& a_{12}=-\left(\hat{g}_{1},\left(p^{2} / 2+E\right)^{-1} \hat{g}_{1, \rho}\right), \\
& a_{22}=\frac{1}{\lambda_{2}}-\left\|\left(p^{2} / 2+E\right)^{-1 / 2} \hat{g}_{1}\right\|^{2}, \\
& b_{11}=-\left(\hat{g}_{1},\left(p^{2} / 2+E\right)^{-1} \hat{g}_{2}\right), \\
& b_{12}=-\left(\hat{g}_{2},\left(p^{2} / 2+E\right)^{-1} \hat{g}_{1, \rho}\right), \\
& b_{21}=-\left(\hat{g}_{2, \rho},\left(p^{2} / 2+E\right)^{-1} \hat{g}_{1}\right), \\
& d_{11}=\frac{1}{\mu_{1}}-\left\|\left(p^{2} / 2+E\right)^{-1 / 2} \hat{g}_{2}\right\|^{2}, \\
& d_{12}=-\left(\hat{g}_{2},\left(p^{2} / 2+E\right)^{-1} \hat{g}_{2, \rho}\right), \\
& d_{22}=\frac{1}{\mu_{2}}-\left\|\left(p^{2} / 2+E\right)^{-1 / 2} \hat{g}_{2}\right\|^{2} .
\end{aligned}
$$

Considerando la hipótesis de que las funciones $\hat{g}_{i}$ son no negativas, y puesto que el operador $\left(-\frac{\Delta}{2}+E\right)^{-1}$ preserva positividad[RS4, pg. 201-203] se tiene

$$
\left(\hat{g}_{i},\left(p^{2} / 2+E\right)^{-1} \hat{g}_{i, \rho}\right)=\left(g_{i},\left(-\frac{\Delta}{2}+E\right)^{-1} g_{i}(x-\rho(t))\right)=\left(\hat{g}_{i, \rho},\left(p^{2} / 2+E\right)^{-1} \hat{g}_{i}\right)
$$

La ecuación anterior justifica el hecho de que $a_{12}$ y $d_{12}$ son números reales. Tambien,

$$
\left(\hat{g}_{2, \rho},\left(p^{2} / 2+E\right)^{-1} \hat{g}_{1, \rho}\right)=\int_{\mathbf{R}^{n}} \overline{e^{i p \cdot \rho(t)}} \overline{\hat{g}}_{2}(p) \frac{e^{i p \cdot \rho(t)} \hat{g}_{1}(p)}{p^{2} / 2+E} d p=\left(\hat{g}_{2},\left(p^{2} / 2+E\right)^{-1} \hat{g}_{1}\right)
$$


De manera que el sistema de ecuaciones formada por (3.7), (3.8), (3.9) y (3.10), se expresa matricialmente como

$$
\left[\begin{array}{cccc}
\lambda_{1} a_{11} & \lambda_{2} a_{12} & \mu_{1} b_{11} & \mu_{2} b_{21} \\
\lambda_{1} a_{12} & \lambda_{2} a_{22} & \mu_{1} b_{12} & \mu_{2} b_{11} \\
\lambda_{1} b_{11} & \lambda_{2} b_{12} & \mu_{1} d_{11} & \mu_{2} d_{12} \\
\lambda_{1} b_{21} & \lambda_{2} b_{11} & \mu_{1} d_{12} & \mu_{2} d_{22}
\end{array}\right]\left[\begin{array}{c}
\left(\hat{g}_{1}, \hat{\psi}\right) \\
\left(\hat{g}_{1, \rho}, \hat{\psi}\right) \\
\left(\hat{g}_{2}, \hat{\psi}\right) \\
\left(\hat{g}_{2, \rho}, \hat{\psi}\right)
\end{array}\right]=0
$$

De esta manera, $-E$ es un valor propio de $H(t)$ si y sólo si, el determinante de la matriz con entradas reales $M$ tiene determinante igual a cero, con

$$
M=\left[\begin{array}{llll}
a_{11} & a_{12} & b_{11} & b_{21} \\
a_{12} & a_{22} & b_{12} & b_{11} \\
b_{11} & b_{12} & d_{11} & d_{12} \\
b_{21} & b_{11} & d_{12} & d_{22}
\end{array}\right]=\left[\begin{array}{ll}
A & B^{T} \\
B & D
\end{array}\right]
$$

Haciendo cálculos se obtiene

$$
\begin{aligned}
\operatorname{det}(M)= & \left(a_{11} a_{22}-a_{12}^{2}\right)\left(d_{11} d_{22}-d_{12}^{2}\right)+\left(b_{11}^{2}-b_{12} b_{21}\right)^{2} \\
& -a_{22}\left(b_{21}^{2} d_{11}-2 b_{11} b_{21} d_{12}+b_{11}^{2} d_{22}\right) \\
& -2 a_{12}\left(-b_{11} b_{21} d_{11}+b_{11}^{2} d_{12}+b_{12} b_{21} d_{12}-b_{11} b_{12} d_{22}\right) \\
& -a_{11}\left(b_{11}^{2} d_{11}-2 b_{11} b_{12} d_{12}+b_{12}^{2} d_{22}\right)
\end{aligned}
$$

Observemos que

$$
\begin{aligned}
\left|b_{21}\right| & =\left|-\int \overline{e^{i p \cdot \rho(t)}} \overline{\hat{g}}_{2}(p) \frac{\hat{g}_{1}(p)}{p^{2} / 2+E} d p\right| \\
& \leq \int\left|\overline{\hat{g}}_{2}(p) \frac{\hat{g}_{1}(p)}{p^{2} / 2+E}\right| d p \\
& =\int \overline{\hat{g}}_{1}(p) \frac{\hat{g}_{2}(p)}{p^{2} / 2+E} d p \\
& =\left(\hat{g}_{1},\left(p^{2} / 2+E\right)^{-1} \hat{g}_{2}\right)=-b_{11} .
\end{aligned}
$$

De manera análoga, se obtiene que

$$
\left|b_{12}\right| \leq-b_{11}
$$

Observemos que, los números $a_{12}, b_{12}, b_{21}$ y $d_{12}$ son negativos pues $g_{i}$ son no negativas y $\left(-\frac{1}{2} \Delta+E\right)^{-1}$ preserva positividad. 
Además, por hipótesis $\lambda_{1}<\lambda_{1}+\lambda_{2}$. Por el corolario 2.1 obtenemos que existe $E_{0}(1)>E_{1}$, valor propio del operador $-\frac{1}{2} \Delta-\left(\lambda_{1}+\lambda_{2}\right) V_{1}$, que cumple

$$
\frac{1}{\lambda_{1}+\lambda_{2}}=\left\|\left(p^{2} / 2+E_{0}(1)\right)^{-1 / 2} \hat{g}_{1}\right\|^{2} .
$$

Entonces, si $E_{0}(1)<E$,

$$
\frac{1}{\lambda_{1}+\lambda_{2}} \geq\left\|\left(p^{2} / 2+E\right)^{-1 / 2} \hat{g}_{1}\right\|^{2}
$$

y por tanto,

$$
a_{i i}=\frac{1}{\lambda_{i}}-\left\|\left(p^{2} / 2+E\right)^{-1 / 2} \hat{g}_{1}\right\|^{2}>0, \quad \text { para } i=1,2 \text { si } E_{0}(1)<E .
$$

También, $E_{0}(2)<E$ en este caso, pues por hipótesis $E_{1}>E_{0}(2)$, así que por analogía se tiene

$$
d_{i i}=\frac{1}{\mu_{i}}-\left\|\left(p^{2} / 2+E\right)^{-1 / 2} \hat{g}_{2}\right\|^{2}>0, \quad \text { para } i=1,2 .
$$

De (3.14), (3.15),(3.16),(3.18) y (3.19) se obtiene, para $E>E_{0}(1)$,

$$
\begin{aligned}
\operatorname{det}(M) \geq & \left(a_{11} a_{22}-a_{12}^{2}\right)\left(d_{11} d_{22}-d_{12}^{2}\right)+\left(b_{11}^{2}-b_{12} b_{21}\right)^{2} \\
& -a_{22} b_{11}^{2}\left(d_{11}-2 d_{12}+d_{22}\right)-2 a_{12} b_{11}^{2}\left(-d_{11}+2 d_{12}-d_{22}\right) \\
& -a_{11} b_{11}^{2}\left(d_{11}-2 d_{12}+d_{22}\right)
\end{aligned}
$$

Considerando que

$$
\begin{array}{r}
a_{22}\left(d_{11}-2 d_{12}+d_{22}\right)+2 a_{12}\left(-d_{11}+2 d_{12}-d_{22}\right)+a_{11}\left(d_{11}-2 d_{12}+d_{22}\right) \\
=\left(a_{22}-2 a_{12}+a_{11}\right)\left(d_{11}-2 d_{12}+d_{22}\right)
\end{array}
$$

y

$$
\left(\hat{g}_{1},\left(p^{2} / 2+E\right)^{-1} \hat{g}_{1, \rho}\right)=\left|\left(\hat{g}_{1},\left(p^{2} / 2+E\right)^{-1} \hat{g}_{1, \rho}\right)\right| \leq\left\|\left(p^{2} / 2+E\right)^{-1 / 2} \hat{g}_{1}\right\|^{2}
$$


se sigue, por (3.20), que

$$
\begin{aligned}
\operatorname{det}(M) \geq & \left(a_{11} a_{22}-a_{12}^{2}\right)\left(d_{11} d_{22}-d_{12}^{2}\right)+\left(b_{11}^{2}-b_{12} b_{21}\right)^{2} \\
& -a_{22} b_{11}^{2}\left(d_{11}-2 d_{12}+d_{22}\right)-2 a_{12} b_{11}^{2}\left(-d_{11}+2 d_{12}-d_{22}\right) \\
& -a_{11} b_{11}^{2}\left(d_{11}-2 d_{12}+d_{22}\right) \\
\geq & \left(a_{11} a_{22}-a_{12}^{2}\right)\left(d_{11} d_{22}-d_{12}^{2}\right)+\left(b_{11}^{2}-b_{12} b_{21}\right)^{2} \\
& -b_{11}^{2}\left(\frac{1}{\lambda_{1}}+\frac{1}{\lambda_{2}}\right)\left(\frac{1}{\mu_{1}}+\frac{1}{\mu_{2}}\right) \\
\geq & \left(a_{11} a_{22}-\left\|\left(p^{2} / 2+E\right)^{-1 / 2} \hat{g}_{1}\right\|^{4}\right)\left(d_{11} d_{22}-\left\|\left(p^{2} / 2+E\right)^{-1 / 2} \hat{g}_{2}\right\|^{4}\right) \\
- & b_{11}^{2}\left(\frac{1}{\lambda_{1}}+\frac{1}{\lambda_{2}}\right)\left(\frac{1}{\mu_{1}}+\frac{1}{\mu_{2}}\right)
\end{aligned}
$$

donde, en la última desigualdad usamos el hecho de que

$$
\begin{aligned}
& a_{11} a_{22}-a_{12}^{2} \geq \frac{1}{\lambda_{1} \lambda_{2}}\left(1-\left(\lambda_{1}+\lambda_{2}\right)\left\|\left(p^{2} / 2+E\right)^{-1 / 2} \hat{g}_{1}\right\|^{2}\right)>0, \text { si } E>E_{0}(1) \\
& d_{11} d_{22}-d_{12}^{2} \geq \frac{1}{\lambda_{1} \lambda_{2}}\left(1-\left(\mu_{1}+\mu_{2}\right)\left\|\left(p^{2} / 2+E\right)^{-1 / 2} \hat{g}_{2}\right\|^{2}\right)>0, \text { si } E>E_{1} .
\end{aligned}
$$

Además, cuando $t=0$ se cumple que $g_{i}=g_{i, \rho}$, para $i=1,2$, pues $\rho(0)=0$, de manera que en este caso la desigualdad (3.20) se convierte en una igualdad. Al simplificar el lado derecho de la desigualdad (3.22) se obtiene

$$
\begin{array}{r}
\frac{1}{\lambda_{1} \lambda_{2} \mu_{1} \mu_{2}}\left(1-\sum_{i=1}^{2} \lambda_{i}\left\|\frac{\hat{g}_{1}}{\left(p^{2} / 2+E\right)^{1 / 2}}\right\|^{2}\right)\left(1-\sum_{i=1}^{2} \mu_{i}\left\|\frac{\hat{g}_{2}}{\left(p^{2} / 2+E\right)^{1 / 2}}\right\|^{2}\right) \\
-b_{11}^{2}\left(\frac{1}{\lambda_{1}}+\frac{1}{\lambda_{2}}\right)\left(\frac{1}{\mu_{1}}+\frac{1}{\mu_{2}}\right) .
\end{array}
$$

Podemos ver a la expresión anterior como una función de $E$. Derivamos respecto de $E$

$$
\begin{array}{r}
\frac{d}{d E}\left(\left\|\left(p^{2} / 2+E\right)^{-1 / 2} \hat{g}_{i}\right\|\right)=\frac{d}{d E} \int \frac{\left(\hat{g}_{i}(p)\right)^{2}}{p^{2}+E} d p=\int \frac{d}{d E}\left(\frac{\left(\hat{g}_{i}(p)\right)^{2}}{p^{2}+E}\right) d p \\
=-\left\|\left(p^{2} / 2+E\right)^{-1} \hat{g}_{i}\right\| .
\end{array}
$$


donde hemos aplicado el teorema de Lebesgue intercambiar la derivada con la integral $[A W]$. De manera similar se obtiene

$$
\frac{d}{d E}\left(b_{11}\right)=\left(\left(p^{2} / 2+E\right)^{-1} \hat{g}_{i},\left(p^{2} / 2+E\right)^{-1} \hat{g}_{2}\right) .
$$

La expresión (3.26) es positiva pues $\hat{g}_{i}$ son funciones positivas. Al derivar la expresión (3.24) se obtiene que ésta es monótona creciente como función de $E$, de manera que por la proposición (2.1) se concluye que es negativa en el intervalo $\left[E_{0}(2), E_{0}(1)\right]$, pues $E \geq E_{0}(1)$ implica

$$
1-\left(\lambda_{1}+\lambda_{2}\right)\left\|\left(p^{2} / 2+E\right)^{-1 / 2} \hat{g}_{1}\right\|^{2} \geq 0
$$

y, de manera similar, $E \leq E_{0}(2)$ implica

$$
1-\left(\mu_{1}+\mu_{2}\right)\left\|\left(p^{2} / 2+E\right)^{-1 / 2} \hat{g}_{2}\right\|^{2} \leq 0 .
$$

Por lo tanto, existe un único valor $E_{0}>E_{0}$ (1) que es una raíz de (3.24). Además, si existe otra raíz $E$ diferente, debe cumplirse que $E<E_{0}(2)$.

Observemos que (3.24) nos da una cota inferior de (3.14), el determinante de $M$. Ahora buscaremos una cota superior.

Considerando que $\hat{g}_{i}, i=1,2$ son funciones no negativas, y del hecho de que $\left(-\frac{1}{2} \Delta+E\right)^{-1}$ es un operador que preserva positividad, se tiene que $\left(p^{2} / 2+E\right)^{-1} \hat{g}_{i}$ y $\left(p^{2} / 2+E\right)^{-1} \hat{g}_{i, \rho}$ son positivos. Por hipótesis, $0<\mu_{1}+\mu_{2}<\lambda_{1}$, de manera que

$$
\left\|\left(p^{2} / 2+E_{1}\right)^{-1 / 2} \hat{g}_{1}\right\|^{2}=\frac{1}{\lambda_{1}}<\frac{1}{\mu_{1}+\mu_{2}}=\left\|\left(p^{2} / 2+E_{0}(2)\right)^{-1 / 2} \hat{g}_{2}\right\|^{2}
$$

Además, si $E>E_{1}$, entonces

$$
\left\|\left(p^{2} / 2+E\right)^{-1 / 2} \hat{g}_{1}\right\|^{2}<\frac{1}{\lambda_{1}} .
$$

Se tiene también (hipótesis) que, $\lambda_{1}>\mu_{1}>\lambda_{2}>\mu_{2}>0$ por lo que, tomando en cuenta las consideraciones previas, $a_{11}, a_{22}, d_{11}$ y $d_{22}$ son positivos si $E>E_{1}$ y, $a_{12}, b_{11}, b_{12}, b_{21}$ y $d_{12}$ son negativos. Por lo tanto, si $E_{1}<E$,

$$
\begin{aligned}
\operatorname{det} M & =\left(a_{11} a_{22}-a_{12}^{2}\right)\left(d_{11} d_{22}-d_{12}^{2}\right)+\left(b_{11}^{2}-b_{12} b_{21}\right)^{2}-\left(a_{11} b_{11}^{2} d_{11}+a_{22} b_{11}^{2} d_{22}\right)-P \\
& \leq\left(a_{11} a_{22}-a_{12}^{2}\right)\left(d_{11} d_{22}-d_{12}^{2}\right)+\left(b_{11}^{2}-b_{12} b_{21}\right)^{2}-\left(a_{11} b_{11}^{2} d_{11}+a_{22} b_{11}^{2} d_{22}\right) \\
& \leq a_{11} a_{22}\left(d_{11} d_{22}-d_{12}^{2}\right)+\left(b_{11}^{2}-b_{12} b_{21}\right)^{2}-\left(a_{11} b_{11}^{2} d_{11}+a_{22} b_{11}^{2} d_{22}\right) \\
& \leq\left(a_{11} d_{11}-b_{11}^{2}\right)\left(a_{22} d_{22}-b_{11}^{2}\right) .
\end{aligned}
$$


donde $P$ es una expresión positiva obtenida de (3.14). La segunda desigualdad se justifica por (3.23).

Observemos que

$$
\begin{aligned}
& a_{22} d_{22}-b_{11}^{2}=f_{22}(E):= \\
& \frac{1}{\lambda_{2} \mu_{2}}\left[\left(1-\lambda_{2}\left\|\left(p^{2} / 2+E\right)^{-1 / 2} \hat{g}_{1}\right\|\right)\left(1-\mu_{2}\left\|\left(p^{2} / 2+E\right)^{-1 / 2} \hat{g}_{2}\right\|\right)-\lambda_{2} \mu_{2} b_{11}^{2}\right]
\end{aligned}
$$

Para $E=E_{1}>E_{0}(2)$ se tiene

$$
\begin{aligned}
f_{22}\left(E_{1}\right) & \geq \frac{1}{\lambda_{2} \mu_{2}}-\frac{1}{\mu_{2}}\left\|\left(p^{2} / 2+E\right)^{-1 / 2} \hat{g}_{1}\right\|^{2}-\frac{1}{\lambda_{2}}\left\|\left(p^{2} / 2+E\right)^{-1 / 2} \hat{g}_{2}\right\|^{2} \\
& \geq \frac{1}{\lambda_{2} \mu_{2}}-\frac{1}{\lambda_{2}\left(\mu_{1}+\mu_{2}\right)}-\frac{1}{\lambda_{1} \mu_{2}} \\
& =\frac{\lambda_{1} \mu_{1}-\lambda_{2}\left(\mu_{1}+\mu_{2}\right)}{\lambda_{2} \lambda_{1} \mu_{2}\left(\mu_{1}+\mu_{2}\right)}>0 .
\end{aligned}
$$

La primera desigualdad se obtiene aplicando la desigualdad de Schwarz,

$$
b_{11}^{2} \leq\left\|\left(p^{2} / 2+E\right)^{-1 / 2} \hat{g}_{1}\right\|^{2}\left\|\left(p^{2} / 2+E\right)^{-1 / 2} \hat{g}_{2}\right\|^{2} .
$$

La segunda porque $E>E_{0}(2)$, que implica

$$
\frac{1}{\mu_{2}}>\frac{1}{\mu_{1}+\mu_{2}}>\left\|\left(p^{2} / 2+E\right)^{-1 / 2} \hat{g}_{2}\right\|^{2}
$$

La última desigualdad en (3.29) se deduce de

$$
\frac{\lambda_{1}}{\mu_{1}+\mu_{2}}>1>\frac{\lambda_{2}}{\mu_{1}}
$$

ya que por hipótesis $\lambda_{1}>\mu_{1}+\mu_{2}>0$ y $\mu_{1}>\lambda_{2}>0$.

Derivando $f_{22}(E)$ respecto a $E$, se tiene que $\frac{d f_{22}(E)}{d E}$ es

$$
\begin{gathered}
\frac{1}{\lambda_{2} \mu_{2}}\left[2 \mu_{2}\left(1-\lambda_{2}\left\|\left(p^{2} / 2+E\right)^{-1 / 2} \hat{g}_{1}\right\|^{2}\right)\left\|\left(p^{2} / 2+E\right)^{-1 / 2} \hat{g}_{2}\right\|\left\|\left(p^{2} / 2+E\right)^{-1} \hat{g}_{2}\right\|\right] \\
+\frac{1}{\lambda_{2} \mu_{2}}\left[2 \lambda_{2}\left(1-\mu_{2}\left\|\left(p^{2} / 2+E\right)^{-1 / 2} \hat{g}_{2}\right\|^{2}\right)\left\|\left(p^{2} / 2+E\right)^{-1 / 2} \hat{g}_{1}\right\|\left\|\left(p^{2} / 2+E\right)^{-1} \hat{g}_{1}\right\|\right] \\
+2\left(\hat{g}_{1},\left(p^{2} / 2+E\right)^{-1} \hat{g}_{2}\right)\left(\hat{g}_{1},\left(p^{2} / 2+E\right)^{-2} \hat{g}_{2}\right)
\end{gathered}
$$


la cual es no negativa si $E \geq E_{1}$, pues en este caso, $\frac{1}{\lambda_{2}}>\left\|\left(p^{2} / 2+E\right)^{-1 / 2} \hat{g}_{1}\right\|^{2}$ y $\frac{1}{\mu_{2}}>\left\|\left(p^{2} / 2+E\right)^{-1 / 2} \hat{g}_{2}\right\|^{2}$. Por tanto, $f_{22}(E)$ es monótona creciente si $E \geq E_{1}$ y, por (3.29), positiva en dicho intervalo. Procediendo de manera similar se obtiene que

$$
\begin{aligned}
f_{11}(E)= & a_{11} d_{11}-b_{11}^{2} \\
= & \left(\frac{1}{\lambda_{1}}-\left\|\left(p^{2} / 2+E\right)^{-1 / 2} \hat{g}_{1}\right\|^{2}\right)\left(\frac{1}{\mu_{1}}-\left\|\left(p^{2} / 2+E\right)^{-1 / 2} \hat{g}_{2}\right\|^{2}\right) \\
& -\left(\hat{g}_{1},\left(p^{2} / 2+E\right)^{-1} \hat{g}_{2}\right)^{2}
\end{aligned}
$$

es una función monótona estrictamente creciente si $E>E_{1}$. Además, si $E \in\left[E_{\mu_{1}}, E_{1}\right]$, entonces

$$
\frac{1}{\mu_{1}} \geq\left\|\left(p^{2} / 2+E\right)^{-1 / 2} \hat{g}_{2}\right\|^{2} \quad \text { y } \quad\left\|\left(p^{2} / 2+E\right)^{-1 / 2} \hat{g}_{1}\right\|^{2} \leq \frac{1}{\lambda_{1}}
$$

por lo que $f_{22}(E)$ es negativa en $\left[E_{\mu_{1}}, E_{1}\right]$ y debe ser cero para un único valor $E_{\infty}>E_{1}$. Esto permite afirmar que

$$
f_{11}(E) f_{22}(E)=\left(a_{11} d_{11}-b_{11}^{2}\right)\left(a_{22} d_{22}-b_{11}^{2}\right)>0 \text { si } E>E_{\infty}
$$

Ahora probaremos que $E_{0}>E_{\infty}$ : Para ello, sustituimos la identidad

$$
\lambda_{1} \mu_{1} b_{11}^{2}\left(E_{\infty}\right)=\left(1-\lambda_{1}\left\|\left(p^{2} / 2+E_{\infty}\right)^{-1 / 2} \hat{g}_{1}\right\|^{2}\right)\left(1-\mu_{1}\left\|\left(p^{2} / 2+E_{\infty}\right)^{-1 / 2} \hat{g}_{2}\right\|^{2}\right)
$$


en la expresión (3.24) y obtenemos

$$
\begin{aligned}
& \left(1-\left(\lambda_{1}+\lambda_{2}\right)\left\|\left(p^{2} / 2+E_{\infty}\right)^{-1 / 2} \hat{g}_{1}\right\|^{2}\right)\left(1-\left(\mu_{1}+\mu_{2}\right)\left\|\left(p^{2} / 2+E_{\infty}\right)^{-1 / 2} \hat{g}_{2}\right\|^{2}\right) \\
& -\left(\lambda_{1}+\lambda_{2}\right)\left(\mu_{1}+\mu_{2}\right) b_{11}\left(E_{\infty}\right)^{2} \\
= & -\lambda_{1}\left\|\left(p^{2} / 2+E_{\infty}\right)^{-1 / 2} \hat{g}_{1}\right\|^{2}-\mu_{1}\left\|\left(p^{2} / 2+E_{\infty}\right)^{-1 / 2} \hat{g}_{2}\right\|^{2} \\
& +\lambda_{1} \mu_{1}\left(\left\|\left(p^{2} / 2+E_{\infty}\right)^{-1 / 2} \hat{g}_{1}\right\|^{2}\left\|\left(p^{2} / 2+E_{\infty}\right)^{-1 / 2} \hat{g}_{2}\right\|^{2}-b_{11}^{2}\left(E_{\infty}\right)\right) \\
& -\lambda_{2}\left\|\left(p^{2} / 2+E_{\infty}\right)^{-1 / 2} \hat{g}_{1}\right\|^{2}-\mu_{2}\left\|\left(p^{2} / 2+E_{\infty}\right)^{-1 / 2} \hat{g}_{2}\right\|^{2} \\
& +\left(\lambda_{1} \mu_{2}+\lambda_{2}\left(\mu_{1}+\mu_{2}\right)\right)\left(\left\|\left(p^{2} / 2+E_{\infty}\right)^{-1 / 2} \hat{g}_{1}\right\|^{2}\left\|\left(p^{2} / 2+E_{\infty}\right)^{-1 / 2} \hat{g}_{2}\right\|^{2}-b_{11}^{2}\left(E_{\infty}\right)\right) \\
= & -\lambda_{2}\left\|\left(p^{2} / 2+E_{\infty}\right)^{-1 / 2} \hat{g}_{1}\right\|^{2}-\mu_{2}\left\|\left(p^{2} / 2+E_{\infty}\right)^{-1 / 2} \hat{g}_{2}\right\|^{2} \\
& +\left(\lambda_{1} \mu_{2}+\lambda_{2}\left(\mu_{1}+\mu_{2}\right)\right) \frac{\lambda_{1}\left\|\left(p^{2} / 2+E_{\infty}\right)^{-1 / 2} \hat{g}_{1}\right\|^{2}+\mu_{1}\left\|\left(p^{2} / 2+E_{\infty}\right)^{-1 / 2} \hat{g}_{2}\right\|^{2}-1}{\lambda_{1} \mu_{1}} \\
= & \frac{\mu_{2}\left(\lambda_{1}+\lambda_{2}\right)}{\mu_{1}}\left(\left\|\left(p^{2} / 2+E_{\infty}\right)^{-1 / 2} \hat{g}_{1}\right\|^{2}-\frac{1}{\lambda_{1}}\right) \\
& +\frac{\lambda_{2}\left(\mu_{1}+\mu_{2}\right)}{\lambda_{1}}\left(\left\|\left(p^{2} / 2+E_{\infty}\right)^{-1 / 2} \hat{g}_{2}\right\|^{2}-\frac{1}{\mu_{1}+\mu_{2}}\right) .
\end{aligned}
$$

La expresión anterior es negativa pues $E_{0}(2)<E_{1}<E_{\infty}$. Esto implica que $E_{\infty}<E_{0}$ pues ya vimos que la expresión (3.24) es positiva si $E>E_{0}$.

Ahora si, probamos el teorema 3.1.

Demostración.

$$
f(E, t):=\operatorname{det} M=\operatorname{det}\left(\begin{array}{cc}
A & B^{T} \\
B & C
\end{array}\right) .
$$

La función $f(E, t)$ es tal que $f(E, 0)$ no cambia de signo en el intervalo $\left[E_{0}(2), E_{0}\right]$ según vimos en el análisis previo pues la expresión (3.24) es precisamente $f(E, 0)$. Además, observamos que

$$
f(E, 0)<0 \text {, si } E_{\infty}<E<E_{0} .
$$

pues las desigualdades en (3.22) se convierten en identidades cuando $t=0 \mathrm{y}$ $f\left(E_{\infty}, 0\right)<0$. Por la proposición 1.2 , se sigue que $a_{12}, b_{12}, b_{21}, d_{12}$ tienden a cero si $t \rightarrow \pm \infty$. Por tanto

$$
f(E, \infty):=\lim _{t \rightarrow \pm \infty} f(E, t)=\left(a_{11} d_{11}-b_{11}^{2}\right)\left(a_{22} d_{22}-b_{11}^{2}\right) .
$$


De la relación (3.31) se desprende que

$$
f(E, \infty)>0, \text { para } E_{\infty}<E<E_{0} .
$$

De (3.33) y (3.35), aplicando el teorema de valor medio del cálculo diferencial, se concluye que existe $t \in \mathbb{R}$ tal que

$$
f(E, t)=0, \quad \text { con } \quad E_{\infty}<E<E_{0}
$$

es decir, si $E \in\left[E_{\infty}, E_{0}\right]$, entonces existe $t \in \mathbb{R}$ tal que $-E$ es valor propio de $H(t)$.

La otra parte del teorema se sigue de (3.22), (3.23) y (3.24) se sigue que $-E$ no puede ser un valor propio de $H(t)$ si $E_{0}<E$ pues para $E>E_{0}$, $f(E, t)>0$. De (3.27) se deduce que

$$
f(E, t) \leq f(E, \infty)<0, \quad \text { si } \quad E_{1} \leq E<E_{\infty},
$$

lo que muestra que en el intervalo $\left(-E_{\infty},-E_{1}\right]$ no existen valores propios para toda $t \in \mathbb{R}$.

\subsection{Resultado principal. La dimensión de la proyección espectral sobre $\left[-E_{0},-E_{\infty}\right]$ y el vector propio correspondiente}

En esta sección enunciamos el resultado principal que hemos obtenido en este trabajo. Éste se refiere a la dimensión del espacio propio correspondiente al valor propio $-E(t)$.

Lema 3.3. La dimensión del espacio correspondiente al valor propio $-E(0)$ es 1.

Demostración. Consideremos los siguientes vectores $\vec{x}=\left\langle x_{1}, x_{2}\right\rangle:=$ $\left\langle\left(\hat{g}_{1}, \hat{\psi}\right),\left(\hat{g}_{1, \rho}, \hat{\psi}\right)\right\rangle^{T}$ y $\vec{y}=\left\langle y_{1}, y_{2}\right\rangle:=\left\langle\left(\hat{g}_{2}, \hat{\psi}\right),\left(\hat{g}_{2, \rho}, \hat{\psi}\right)\right\rangle^{T}$. De esta manera, (3.13) puede expresarse como

$$
\left(\begin{array}{cc}
A & B^{T} \\
B & D
\end{array}\right)\left(\begin{array}{l}
\vec{x} \\
\vec{y}
\end{array}\right)=\left(\begin{array}{c}
\overrightarrow{0} \\
\overrightarrow{0}
\end{array}\right)
$$


El sistema anterior es equivalente a

$$
\begin{array}{r}
A \vec{x}+B^{T} \vec{y}=\overrightarrow{0} \\
B \vec{x}+D \vec{y}=\overrightarrow{0}
\end{array}
$$

Observemos que si $E_{0}(2)<E$, entonces

$$
\begin{aligned}
\operatorname{det}(D) & =\left(\frac{1}{\mu_{1}}-\left\|\left(p^{2} / 2+E\right)^{-1 / 2} \hat{g}_{2}\right\|^{2}\right)\left(\frac{1}{\mu_{2}}-\left\|\left(p^{2} / 2+E\right)^{-1 / 2} \hat{g}_{2}\right\|^{2}\right) \\
& -\left(\hat{g}_{2},\left(p^{2} / 2+E\right)^{-1} \hat{g}_{2, \rho}\right)^{2} \\
& =\frac{1}{\mu_{1} \mu_{2}}-\frac{\mu_{1}+\mu_{2}}{\mu_{1} \mu_{2}}\left\|\left(p^{2} / 2+E\right)^{-1 / 2} \hat{g}_{2}\right\|^{2}+\left\|\left(p^{2} / 2+E\right)^{-1 / 2} \hat{g}_{2}\right\|^{4} \\
& -\left(\hat{g}_{2},\left(p^{2} / 2+E\right)^{-1} \hat{g}_{2, \rho}\right)^{2} \\
& \geq \frac{1}{\mu_{1} \mu_{2}}-\frac{\mu_{1}+\mu_{2}}{\mu_{1} \mu_{2}}\left\|\left(p^{2} / 2+E\right)^{-1 / 2} \hat{g}_{2}\right\|^{2} \\
& =\frac{\mu_{1}+\mu_{2}}{\mu_{1} \mu_{2}}\left(\frac{1}{\mu_{1}+\mu_{2}}-\left\|\left(p^{2} / 2+E\right)^{-1 / 2} \hat{g}_{2}\right\|^{2}\right)>0
\end{aligned}
$$

Hemos usado, para justificar la desigualdad, el hecho de que

$$
\left|\left(\hat{g}_{2},\left(p^{2} / 2+E\right)^{-1} \hat{g}_{2, \rho}\right)\right| \leq\left\|\left(p^{2} / 2+E\right)^{-1 / 2} \hat{g}_{2}\right\|^{2}
$$

- la cual es obtenida por la desigualdad de Schwarz y que a su vez implica

$$
0 \leq-\left(\hat{g}_{2},\left(p^{2} / 2+E\right)^{-1} \hat{g}_{2, \rho}\right)^{2}+\left\|\left(p^{2} / 2+E\right)^{-1 / 2} \hat{g}_{2}\right\|^{4}
$$

Esto significa que para $E \in\left[E_{\infty}, E_{0}\right]$, la matriz inversa de $D, D^{-1}$, existe.

De (3.38) se sigue que

$$
\vec{y}=-D^{-1} B \vec{x}
$$

por lo que

$$
\left(A-B^{T} D^{-1} B\right) \vec{x}=\overrightarrow{0}
$$

Hagamos $C:=A-B^{T} D^{-1} B$. Se tiene que $C$ es una matriz simétrica pues $A$ y $D^{-1}$ lo son, de manera que tiene la forma siguiente

$$
C:=\left(\begin{array}{ll}
c_{11} & c_{12} \\
c_{12} & c_{22}
\end{array}\right)
$$


donde

$$
\begin{aligned}
& c_{11}=a_{11}-(\operatorname{det} D)^{-1}\left(b_{11}^{2} d_{22}-2 b_{11} b_{21} d_{12}+b_{21}^{2} d_{11}\right) \\
& c_{12}=a_{12}-(\operatorname{det} D)^{-1}\left(b_{11} b_{12} d_{22}+b_{11} b_{21} d_{11}-b_{11}^{2} d_{12}-b_{12} b_{21} d_{12}\right) \\
& c_{22}=a_{22}-(\operatorname{det} D)^{-1}\left(b_{11}^{2} d_{11}-2 b_{11} b_{12} d_{12}+b_{21}^{2} d_{22}\right)
\end{aligned}
$$

Además, $\operatorname{det} C=0$ pues $-E$ es valor propio de $H(t)$, por lo que los renglones de $C$ son múltiplos, es decir,

$$
C=\left(\begin{array}{cc}
c_{11} & c_{12} \\
k c_{11} & k c_{12}
\end{array}\right)
$$

para alguna constante $k$.

Observemos que la matriz $C$ es no nula cuando $t=0$. En efecto, el valor propio correspondiente $-E_{0}$ cumple

$$
\begin{aligned}
& a_{12}=-\left\|\left(p^{2} / 2+E_{0}\right)^{-1 / 2} \hat{g}_{1}\right\|^{2} \\
& d_{12}=-\left\|\left(p^{2} / 2+E_{0}\right)^{-1 / 2} \hat{g}_{2}\right\|^{2} \\
& b_{21}=b_{12}=b_{11}=-\left(\hat{g}_{1},\left(p^{2} / 2+E_{0}\right)^{-1} \hat{g}_{2}\right) .
\end{aligned}
$$

Así, para este caso se tiene que

$$
\begin{aligned}
c_{12} & =a_{12}-\frac{b_{11}^{2}\left(d_{11}+d_{22}\right)-2 d_{12} b_{11}}{\operatorname{det} D} \\
& =-\left\|\left(p^{2} / 2+E_{0}\right)^{-1 / 2} \hat{g}_{1}\right\|^{2} \\
& -\frac{\left(\hat{g}_{1},\left(p^{2} / 2+E_{0}\right)^{-1} \hat{g}_{2}\right)^{2}\left(\frac{\mu_{1}+\mu_{2}}{\mu_{1} \mu_{2}}-2\left\|\left(p^{2} / 2+E_{0}\right)^{-1 / 2} \hat{g}_{2}\right\|^{2}\right)}{\operatorname{det} D} \\
& -\frac{2\left\|\left(p^{2} / 2+E_{0}\right)^{-1 / 2} \hat{g}_{2}\right\|^{2}\left(\hat{g}_{1},\left(p^{2} / 2+E_{0}\right)^{-1} \hat{g}_{2}\right)^{2}}{\operatorname{det} D} \\
& <0
\end{aligned}
$$

Para justificar la desigualdad en (3.44) usamos el hecho de que

$$
\left\|\left(p^{2} / 2+E\right)^{-1 / 2} \hat{g}_{2}\right\|^{2}<\frac{\mu_{1}+\mu_{2}}{2 \mu_{1} \mu_{2}},
$$

la cual se debe a que $E_{0}>E_{\mu_{1}}$ y $E_{0}>E_{0}(2)$ (se ha probado ya) y ésto implica que 
(i) $\operatorname{det}(D)>0$;

(ii) $\left\|\left(p^{2} / 2+E\right)^{-1 / 2} \hat{g}_{2}\right\|^{2}<\frac{1}{\mu_{1}}<\frac{\mu_{1}+\mu_{2}}{2 \mu_{1} \mu_{2}}$.

Ahora, de (3.41), (3.43) y usando ya el hecho de que $c_{12} \neq 0$, se tiene que $x_{2}=-\frac{c_{11}}{c_{12}} x_{1}$, es decir,

$$
\left(\hat{g}_{1, \rho}, \hat{\psi}\right)=-\frac{c_{11}}{c_{12}}\left(\hat{g}_{1}, \hat{\psi}\right)
$$

De (3.40) se deduce que

$$
\left(\begin{array}{l}
y_{1} \\
y_{2}
\end{array}\right)=-\frac{1}{\operatorname{det} D}\left(\begin{array}{cc}
d_{22} & -d_{12} \\
-d_{12} & d_{11}
\end{array}\right)\left(\begin{array}{ll}
b_{11} & b_{12} \\
b_{21} & b_{22}
\end{array}\right)\left(\begin{array}{l}
x_{1} \\
x_{2}
\end{array}\right)
$$

de donde obtenemos (considerando también (3.45)) las siguientes expresiones

$$
\begin{aligned}
\left(\hat{g}_{2}, \hat{\psi}\right) & =-\frac{b_{11} d_{22}-b_{21} d_{12}}{\operatorname{det} D}\left(\hat{g}_{1}, \hat{\psi}\right)+\frac{c_{11}\left(b_{12} d_{22}-b_{22} d_{12}\right)}{c_{12} \operatorname{det} D}\left(\hat{g}_{1}, \hat{\psi}\right) \\
& =\frac{\left(c_{12} b_{21} d_{12}+c_{11} b_{12} d_{22}\right)-\left(c_{12} b_{11} d_{22}+c_{11} b_{22} d_{12}\right)}{c_{12} \operatorname{det} D}\left(\hat{g}_{1}, \hat{\psi}\right) \\
\left(\hat{g}_{2, \rho}, \hat{\psi}\right) & =-\frac{b_{21} d_{11}-b_{11} d_{12}}{\operatorname{det} D}\left(\hat{g}_{1}, \hat{\psi}\right)+\frac{c_{11}\left(b_{22} d_{11}-b_{12} d_{12}\right)}{c_{12} \operatorname{det} D}\left(\hat{g}_{1}, \hat{\psi}\right) \\
& =\frac{\left(c_{12} b_{11} d_{12}+c_{11} b_{22} d_{11}\right)-\left(c_{12} b_{21} d_{11}+c_{11} b_{12} d_{12}\right)}{c_{12} \operatorname{det} D}\left(\hat{g}_{1}, \hat{\psi}\right) .
\end{aligned}
$$

Sustituimos (3.45), (3.46) y (3.47) en (3.6) para obtener (puesto que la constante $\left.\left(\hat{g}_{1}, \hat{\psi}\right) \neq 0\right)$

$$
\hat{\psi}=\lambda_{1} \frac{\hat{g}_{1}}{\frac{p^{2}}{2}+E}+\lambda_{2} k_{1} \frac{\hat{g}_{1, \rho}}{\frac{p^{2}}{2}+E}+\mu_{1} k_{2} \frac{\hat{g}_{2}}{\frac{p^{2}}{2}+E}+\mu_{2} k_{3} \frac{\hat{g}_{2, \rho}}{\frac{p^{2}}{2}+E} .
$$

donde

$$
\begin{aligned}
& k_{1}=-\frac{c_{11}}{c_{12}} \\
& k_{2}=\frac{\left(c_{12} b_{21} d_{12}+c_{11} b_{12} d_{22}\right)-\left(c_{12} b_{11} d_{22}+c_{11} b_{22} d_{12}\right)}{c_{12} \operatorname{det} D} \\
& k_{3}=\frac{\left(c_{12} b_{11} d_{12}+c_{11} b_{22} d_{11}\right)-\left(c_{12} b_{21} d_{11}+c_{11} b_{12} d_{12}\right)}{c_{12} \operatorname{det} D} .
\end{aligned}
$$

Tomando transformada de Fourier en (3.48) se obtiene el valor propio correspondiente $\psi(0)$. 
Teorema 3.2. El espacio correspondiente al vector propio $-E(t)$ del operador $H(t)$ es unidimensional, para cada $t \in \mathbb{R}$.

Demostración. Probamos que en $t=0$, el espacio correspondiente a $-E(0)$ es de dimensión 1. Aplicando nuevamente el teorema (1.9) usado en la demostración del teorema (2.1), concluimos que el espacio correspondiente a $-E(t)$ es también de dimensión 1 , para cada $t \in \mathbb{R}$.

Finalizamos éste capítulo con la siguiente observación.

Observación. El teorema anterior garantiza que la matriz $C$ (3.43) es no nula, para cada $t \in \mathbb{R}$. En este caso, tomando la entrada no nula para despejar $x$, se obtiene una expresión para el vector propio $\psi(t)$. 


\section{Conclusiones}

En nuestro trabajo hemos generalizado resultados previamente obtenidos para Hamiltonianos dependientes del tiempo, donde se caracteriza al eigenvalor asociado al estado fundamental. El mérito fudamental del trabajo ha sido probar que el Hamiltoniano preserva la misma propiedad sobre la dimensión del proyector espectral sobre el espectro discreto del Hamiltoniano.

No fué posible ofrecer una forma explícita de los estados fundamentales para el Hamiltoniano estudiado en el capítulo 3, a diferencia del estudiado en el capítulo 2, debido a la complejidad de los cálculos.

Las propiedades del Hamiltoniano que hemos probado se utilizan para comprobar a partir de primeros principios el famoso criterio de Massey en física experimental que afirma:

Una transición de estado es improbable, si $\hbar v / d \ll 1$.

donde $d$ es el rango de la interacción y $v$ es la velocidad relativa entre las partículas y el proceso de dispersión.

Sería deseable poder extender estos resultados para Hamiltonianos más generales, por ejemplo con potenciales $V_{i}$ a traza. Se espera que ciertas propiedades se preserven y en las demostraciones puedan usarse alguna de las técnicas usadas en éste trabajo. También se espera que los cálculos sean mucho más complejos. 


\section{Bibliografía}

[AJS] Amrein, W., Jauch, J. y Sinha, B.: Scattering Theory in Quantum Mechanics. Physical Principles an Mathematical Methods, W. A. Benjamin, Inc. Massachusetts, 1977.

[Arr1] Arredondo, J. H.: Asymtotic transition probabilities. J. Math. Phys. 30(10) (1989), 2291-2296.

[Arr2] Arredondo, J.H.: Teoría de operadores con Aplicaciones a la Física. Publicaciones UAM-I. México, 1997.

[Arr3] Arredondo, J.H.: Comunicaciones Técnicas. Serie Naranja, IIMASUNAM, Vol. 459, 1987.

[AS] Arredondo, J. H. y Seibert, P.: Characterization of the fundamental state eigenvalue for some time dependent Hamiltonians. Aportaciones Matemáticas. Serie comunicaciones 29 (2001), 3-10.

[AW] Arredondo, J. H. y Wawrczñcyk, A.: Medidas e Integrales Funcionales. Notas de curso. Publicaciones UAM-I, México, 2004.

[Do] Donoghue, W. F.: Distributions and Fourier Transforms, Academic Press, New York, 1969.

[HS] Hislop, P.D. y Sigal, I. M.: Introduction to Spectral Theory: with applications to Schrödinger operators, Springer-Verlag, New York, 1996.

[Ka] Kato, T.: Perturbation Theory for Linear Operators, segunda edición, Springer-Verlag, New York, 1980.

[LL] Lieb, E.H. y Loss, M.: Anulysis, Graduate Studies in Mathematics, Vol. 14. American Mathematical Society, 1996. 
[RS1] Reed, M. y Simon, B.: Methods of Modern Mathematical Physics, I. Functional Analysis. Academic Press, New York, 1978.

[RS2] Reed, M. y Simon, B.: Methods of Modern Mathematical Physics, II. Fourier Analysis, Self-Adjointness, Academic Press, New York, 1978.

[RS3] Reed, M. y Simon, B.: Methods of Modern Mathematical Physics, III. Scattering Theory, Academic Press, New York, 1978.

[RS4] Reed, M. y Simon, B.: Methods of Modern Mathematical Physics, IV. Analysis of Operators, Academic Press, New York, 1978.

[We] Weidmann, J.: Linear Operators in Hilbert Spaces, Springer-Verlag, New York, 1980.

[Yo] Yosida, K.: Functional Analysis, Springer-Verlag, Berlin, 1980. 